\title{
Super-Eddington accretion and feedback from the first massive seed black holes
}

\author{
John A. Regan ${ }^{\oplus},{ }^{\star} \dagger$ Turlough P. Downes, ${ }^{1}$ Marta Volonteri, ${ }^{2}$ Ricarda Beckmann ${ }^{\oplus}, 2$ \\ Alessandro Lupi ${ }^{\oplus},{ }^{2}$ Maxime Trebitsch ${ }^{\oplus 2}$ and Yohan Dubois ${ }^{2}$ \\ ${ }^{1}$ Centre for Astrophysics \& Relativity, School of Mathematical Sciences, Dublin City University, Glasnevin, D09 W6Y4, Ireland \\ ${ }^{2}$ Sorbonne Universites, UPMC Univ Paris 6 et CNRS, UMR 7095, Institut d'Astrophysique de Paris, 98 bis bd Arago, F-75014 Paris, France
}

Accepted 2019 April 8. Received 2019 April 8; in original form 2018 November 12

\begin{abstract}
Super-Eddington accretion on to massive black hole seeds may be commonplace in the early Universe, where the conditions exist for rapid accretion. Direct-collapse black holes are often invoked as a possible solution to the observation of supermassive black holes (SMBHs) in the pre-reionization Universe. We investigate here how feedback, mainly in the form of bipolar jets, from super-Eddington accreting seed black holes will affect their subsequent growth. We find that, nearly independently of the mass loading of the bipolar jets, the violent outflows generated by the jets evacuate a region of approximately $0.1 \mathrm{pc}$ surrounding the black hole seed. However, the jet outflows are unable to break free of the halo and their impact is limited to the immediate vicinity of the black hole. The outflows suppress any accretion for approximately a dynamical time. The gas then cools, recombines, and falls back to the centre, where high accretion rates are again observed. The overall effect is to create an effective accretion rate with values of between 0.1 and 0.5 times the Eddington rate. If this episodic accretion rate is maintained for order 500 million years, then the black hole will increase in mass by a factor of between 3 and 300 but far short of the factor of $10^{4}$ required for the seeds to become the SMBHs observed at $z>6$. Therefore, direct-collapse black holes born into atomic cooling haloes and which experience strong negative mechanical feedback will require external influences (e.g. rapid major mergers with other haloes) to promote efficient accretion and reach SMBH masses within a few hundred million years.
\end{abstract}

Key words: methods: numerical - large-scale structure of Universe-cosmology: theory.

\section{INTRODUCTION}

The discovery of supermassive black holes (SMBHs) with masses in excess of $10^{9} \mathrm{M}_{\odot}$ at redshifts greater than $z=6$ (Fan et al. 2006; Mortlock et al. 2011; Tang et al. 2019) presents a significant difficulty for theories of black hole formation and growth. Black holes are expected to form as the end point of massive stars. Black holes forming from the first generation of massive Population III (Pop III) stars have initial seed masses close to their final stellar mass (e.g. Woosley, Heger \& Weaver 2002). However, these Pop III remnant black holes are expected to be born 'starving' (Whalen, Abel \& Norman 2004; O’Shea et al. 2005; Wang, Chen \& Hu 2006; Johnson \& Bromm 2007; Alvarez, Wise \& Abel 2009; Milosavljević, Couch \& Bromm 2009; Jeon et al. 2012). A more recent study by Smith et al. (2018) using a sample of approximately

^E-mail: john.regan@dcu.ie

$\dagger$ Marie Skłodowska-Curie Fellow.
15000 Pop III remnant black holes from the Renaissance simulation suite (Xu, Wise \& Norman 2013; Chen et al. 2014; O'Shea et al. 2015) saw no evidence for significant accretion on to the remnant black holes, with Pop III remnants increasing their mass by at most 10 percent over several hundred million years. ${ }^{1}$ The black holes are typically born into low-density environments due to an initial supernova explosion, which results in severely stunted growth. For Pop III stars within the direct-collapse window (Heger et al. 2003) the black hole initially experiences rapid accretion; however, the phase is short-lived, with high-density gas quickly consumed by further star formation. Even if Pop III remnant stars can remain in a region of high density, where local star formation is suppressed, a Pop III remnant would need to accrete at the Eddington limit for several hundred megayears in order to reach a mass of close to

\footnotetext{
${ }^{1}$ It should be noted that this study investigated the accretion on to the black holes in post-processing only and neglected the impact of dynamical friction, which may have increased the accretion rates.
} 
a billion solar masses by a redshift of 6 . Such a scenario is very unlikely based on current research.

In light of this, several other pathways have been explored to attempt to understand the appearance of SMBHs in the first billion years of the Universe. The scenarios have broadly been divided into light seed scenarios and heavy seed scenarios. Light seed scenarios encompass mechanisms where the initial black hole mass is 'light' $\left(M_{\text {init }} \sim 100 \mathrm{M}_{\odot}\right)$ but grows rapidly. The Pop III remnant case falls under the light seed scenarios, as do cases where initially light seeds rapidly merge together to form a more massive object. Several authors have considered a scenario where stellar collisions in high-redshift, dense star clusters lead to the runaway growth of a single star (Gürkan, Freitag \& Rasio 2004; Portegies Zwart et al. 2004; Freitag, Gürkan \& Rasio 2006; Gürkan, Fregeau \& Rasio 2006; Omukai, Schneider \& Haiman 2008; Devecchi \& Volonteri 2009; Katz, Sijacki \& Haehnelt 2015; Habouzit, Volonteri \& Dubois 2017). In this scenario, a dense stellar cluster becomes unstable to gravitational collapse, leading to the merger of a significant number of the stars in the cluster and the formation of a single massive star through mass segregation. The most massive stars that emerge from the cluster are expected to have initial masses of the order of $1000 \mathrm{M}_{\odot}$. Alternatively, there are a number of scenarios where a heavy seed $\left(M_{\text {init }} \gtrsim 10^{4} \mathrm{M}_{\odot}\right)$ may emerge. In the centre of rapidly accreting atomic cooling haloes, which are metal-free, a supermassive star (SMS) is expected to form (Eisenstein \& Loeb 1995; Bromm \& Loeb 2003; Regan \& Haehnelt 2009a,b). SMS formation requires very high accretion rates in excess of $0.01 \mathrm{M}_{\odot} \mathrm{yr}^{-1}$ (Begelman, Volonteri \& Rees 2006; Begelman, Rossi \& Armitage 2008; Schleicher et al. 2013; Sakurai et al. 2016b) to inflate the envelope around the protostar and sustain a supermassive (or possibly a quasi-) star (Hosokawa, Omukai \& Yorke 2013a; Hosokawa et al. 2013b; Inayoshi, Omukai \& Tasker 2014; Umeda et al. 2016; Woods et al. 2017; Haemmerlé et al. $2018 \mathrm{a}, \mathrm{b})$. If accretion rates in excess of $0.01 \mathrm{M}_{\odot} \mathrm{yr}^{-1}$ can be sustained for the lifetime of the star, then the star is expected to collapse into a massive black hole seed at the end of its lifetime, either through the general relativistic instability (Chandrasekhar 1964) or after the star runs out of nuclear fuel. The final mass of the SMS is expected to be well in excess of $10^{4} \mathrm{M}_{\odot}$. The collapse into a direct-collapse black hole then leaves a black hole seed with a large initial mass. If no supernova explosion occurs, then the black hole can be born into a region with a plentiful supply of gas from which it can accrete.

Typically, the environmental conditions required for the heavy seed model require strong sources of nearby Lyman-Werner radiation, which can efficiently dissociate $\mathrm{H}_{2}$ (Dijkstra et al. 2008; Dijkstra, Ferrara \& Mesinger 2014; Visbal, Haiman \& Bryan 2014; Regan et al. 2017). However, dynamical processes that collisionally dissociate $\mathrm{H}_{2}$ may also induce the correct environmental conditions for direct-collapse black holes (Mayer et al. 2010; Inayoshi \& Omukai 2012; Fernandez et al. 2014; Inayoshi, Visbal \& Kashiyama 2015; Mayer et al. 2015). Similarly, relative streaming velocities between baryons and dark matter following recombination (Tseliakhovich \& Hirata 2010) have been investigated by several authors in the context of the first massive black holes (Tanaka \& Li 2014; Hirano et al. 2017; Schauer et al. 2017), with promising results. In summary, several pathways remain open to generating environmental conditions for the formation of massive back hole seeds.

Accretion on to the black hole in either scenario will determine the future growth of the black hole. The Eddington accretion rate can be derived by equating the gravitational force of a black hole to the radiative force experienced by the infalling matter. The resulting force balance applies in the case of a spherically symmetric collapse, with the Eddington accretion rate given by

$\dot{M}_{\mathrm{Edd}}=\frac{4 \pi G M_{\mathrm{BH}} m_{\mathrm{p}}}{\eta \sigma_{\mathrm{T}} c}$,

where $M_{\mathrm{BH}}$ is the black hole mass, $m_{\mathrm{p}}$ is the proton mass, $\eta$ is the radiative efficiency, $\sigma_{\mathrm{T}}$ is the Thomson scattering crosssection, and $c$ is the speed of light. However, it is known that in non-spherically symmetric circumstances, the Eddington rate can be breached and super-Eddington accretion may persist. In this case, accretion can then proceed extremely rapidly. Numerous models of super-Eddington accretion exist. For example, the slim disc model of super-Eddington accretion was originally developed by Abramowicz et al. (1988) to investigate scenarios where the Eddington limitation could be broken. Super-Eddington models of accretion on to stellar mass black holes have recently been investigated by a number of authors (Sagdowski 2009; Sa̧dowski et al. 2014; Sạdowski et al. 2016; Sa̧dowski \& Narayan 2016; Jiang, Stone \& Davis 2017) with results consistently showing that super-Eddington accretion can be achieved, with observational evidence also mounting to support super-Eddington accretion (e.g. Du et al. 2018).

Super-Eddington accretion has been shown, through numerical models, to generate powerful bipolar jets, which become active as the accretion rate exceeds the Eddington rate. These jets, though highly collimated, have the potential to shut off the very accretion flow that is driving the jets, and regulate the accretion flow to values sub-Eddington. Previous investigations have included only radiative feedback from $\mathrm{BH}$ seeds accreting at super-Eddington rates (Pacucci, Volonteri \& Ferrara 2015; Inayoshi, Haiman \& Ostriker 2016; Lupi et al. 2016; Pezzulli, Valiante \& Schneider 2016; Sakurai, Inayoshi \& Haiman 2016a; Pezzulli et al. 2017; Sugimura et al. 2017; Toyouchi et al. 2019). Furthermore, the works listed above have been, by necessity, somewhat idealized. We investigate here a self-consistent $3 \mathrm{D}$ cosmological setting where an embryonic black hole seed finds itself at the centre of a strong accretion flow. We investigate if an initial seed mass black hole accreting above the Eddington rate can sustain a large accretion in the presence of bipolar jets.

\section{NUMERICAL FRAMEWORK}

In this study we have used the publicly available adaptive mesh refinement code $\mathrm{ENZO}^{2}$ to study the birth of a massive black hole seed from an SMS. We have utilized the SMARTSTAR particles introduced in Regan \& Downes (2018a) and augmented them with subgrid prescriptions specific to a black hole seed as we now discuss.

\subsection{ENZO}

ENZO $^{3}$ (Bryan et al. 2014) is an adaptive mesh refinement code ideally suited to simulations of the high-redshift universe. Gravity in ENZO is solved using a fast Fourier technique (Hockney \& Eastwood 1988), which solves the Poisson equation on the root grid at each time-step. On subgrids, the boundary conditions are interpolated to the subgrids and the Poisson equation is then solved at each time-step. Dark matter is represented using particles, with

\footnotetext{
${ }^{2}$ http://enzo-project.org/

${ }^{3}$ Changeset:48882af312bc
} 
each particle stored on the highest refinement grid available to it and thus the particle has the same time-step as the gas on that grid. The particle densities are interpolated using the cloud-incell technique on to the grid and solved at the same time as the gas potential. ENZO contains several hydrodynamics schemes to solve the Euler equation. To model the physics of jet launching, we use the ZEUS hydrodynamic solver (Stone \& Norman 1992a,b). A known limitation of the ZEUS solver is the inclusion of artificial viscosity that can cause spurious heating of gas upstream from a shock front (Anninos \& Norman 1994). However, the correct Rankine-Hugoniot jump conditions are none the less achieved. The very high resolution of our simulations, and in particular the small number of cells over which jets are launched, goes some way towards mitigating these effects. Furthermore, the ZEUS solver is very robust and able to follow the sharp discontinuities that arise as the jets are launched. ZEUS is second-order accurate in space and first-order accurate in time.

Chemistry is an important component in following the collapse of (ideal) gas. We use the Grackle $e^{4,5}$ (Smith et al. 2017) library to follow the evolution of ten individual species: $\mathrm{H}, \mathrm{H}^{+}, \mathrm{He}, \mathrm{He}^{+}$, $\mathrm{He}^{++}, \mathrm{e}^{-}, \mathrm{H}_{2}, \mathrm{H}_{2}^{+} \mathrm{H}^{-}$, and $\mathrm{HeH}^{+}$. We adopt here the 26-reaction network determined by Glover (2015a) as the most appropriate network for solving the chemical equations required by gas of primordial composition with no metal pollution and exposed to an external radiation source. The network includes the most up-todate rates as described in Glover \& Jappsen (2007), Glover \& Abel (2008), Glover \& Savin (2009), Coppola et al. (2011), Coppola et al. (2012), Glover (2015a), Glover (2015b), and Latif et al. (2015). The cooling mechanisms included in the model are collisional excitation cooling, collisional ionization cooling, recombination cooling, bremsstrahlung, and Compton cooling off the cosmic microwave background. $\mathrm{H}_{2}$ line cooling is explicitly followed as part of the Grackle chemistry network following the prescription given by Abel et al. (1997).

\subsection{Simulation set-up}

The simulation explored here is the 'Ref20_100J21_OT' simulation from Regan \& Downes (2018b; hereafter R18b). This simulation used a Lyman-Werner background of $100 J_{21}$ to dissociate $\mathrm{H}_{2}$ and allowed for the formation of an atomic cooling halo in which an SMS can form. The SMS formed at a redshift of $z=24.7$. The maximum resolution of the simulation was set to $2.5 \times 10^{-4} \mathrm{pc}(\sim 50 \mathrm{au})$. At this resolution, resolving the outer envelope of the SMS becomes possible. In R18b the simulation was run for $250 \mathrm{kyr}$, at which point a single SMS was accreting at approximately $10^{-2} \mathrm{M}_{\odot} \mathrm{yr}^{-1}$ (see fig. 2 from R18b) and had achieved a mass of approximately $15000 \mathrm{M}_{\odot}$. We begin the simulation for this study from this point. We briefly review the original simulation for completeness.

The original simulation was run within a cosmological box of $2 h^{-1} \mathrm{Mpc}$ (comoving), on a root grid of $256^{3}$ and with three levels of nested grids. The grid nesting and initial conditions were created using MUSIC (Hahn \& Abel 2011). Within the most refined region (i.e. level 3) the dark matter particle mass is $\sim 103 \mathrm{M}_{\odot}$. In order to further increase the dark matter resolution of our simulations, we split the dark matter particles according to the prescription of Kitsionas \& Whitworth (2002), as described in Regan, Johansson \& Wise (2015). We split particles centred on the position of the final

\footnotetext{
${ }^{4}$ https://grackle.readthedocs.org/

${ }^{5}$ Changeset: $482876 \mathrm{c} 71 \mathrm{f} 73$
}

collapse as found from lower resolution simulations within a region with a comoving side length of $43.75 h^{-1} \mathrm{kpc}$. Each particle is split into 13 daughter particles, resulting in a final high-resolution region with a dark matter particle mass of $\sim 8 \mathrm{M}_{\odot}$. The particle splitting is done at a redshift of 40 , well before the collapse of the target halo. Convergence testing to study the impact of lower dark matter particle masses was discussed in Regan et al. (2015).

The baryon resolution is set by the size of the grid cells. In the highest resolution region this corresponds to approximately $0.48 h^{-1} \mathrm{kpc}$ comoving (before adaptive refinement). Setting the maximum refinement level for this simulation to 20 results in a maximum resolution of $2.5 \times 10^{-4} \mathrm{pc}$. As is standard in simulations of this type, refinement is triggered in ENZO when certain user defined thresholds are exceeded. The refinement criteria used in this work were based on three physical measurements: (1) the dark matter particle overdensity, (2) the baryon overdensity, and (3) the Jeans length. The first two criteria introduce additional meshes when the overdensity of a grid cell with respect to the mean gas or dark matter density exceeds 8.0. Furthermore, we set the MinimumMassForRefinementExponent parameter to -0.1, making the refinement more aggressive for the baryon and dark matter overdensity and hence making the behaviour of the adaptive mesh 'super-Lagrangian' in nature (see Bryan et al. 2014 for further details). This technique also reduces the threshold for refinement as higher densities are reached. For the final criteria we set the number of cells per Jeans length to be 32 in these runs.

In order to suppress Pop III star formation and allow the simulation to form pristine atomic cooling haloes, we imposed an artificial Lyman-Werner background. We set the effective temperature of the background radiation field to $T_{\text {eff }}=30000 \mathrm{~K}$. This background temperature suitably models the spectrum of a population of young stars (Wolcott-Green \& Haiman 2012; Sugimura, Omukai \& Inoue 2014; Latif et al. 2015). The effective temperature of the background is important as the radiation temperature determines the dominant photodissociation reaction set in the irradiated halo. This in turn leads to a value of $J_{\text {crit }}$ - the flux above which complete isothermal collapse of the irradiated halo is observed due to the complete suppression of $\mathrm{H}_{2}$.

As the gas density increases in high-density regions, hydro codes, including ENZO, require a method to convert the high-density gas into stars in many cases. This is done to deal with gas that has reached the maximum allowed refinement level of the simulation and for which further collapse is being artificially suppressed through artificial pressure support. Within 'Ref20_100J21_OT', simulation particles were introduced once the following criteria were met.

(i) The cell is at the highest refinement level.

(ii) The cell exceeds the Jeans density.

(iii) The flow around the cell is converging along all axes.

(iv) The cooling time of the cell is less than the freefall time.

(v) The cell is at a local minimum of the gravitational potential.

As described in R18b, all 'stars' that form are initially assumed to be stars with low surface temperatures that are appropriate for main-sequence SMSs and less massive proto-stars on the Hayashi track. As long as the accretion rate remains above a critical value of $\dot{M}_{*} \gtrsim 0.04 \mathrm{M}_{\odot} \mathrm{yr}^{-1}$ (Sakurai et al. 2016b), the star remains an SMS. If the accretion rate drops below this critical value, the star contracts and becomes a Pop III star. In 'Ref20_100J21_OT', the accretion rate dropped below the critical value shortly after formation, after approximately $25 \mathrm{kyr}$. None the less, the accretion rate remained high even though the ionizing radiation from the 
Pop III was able to ionize and heat some of the gas immediately surrounding the proto-star. Similar results were observed in the simulations of Chon, Hosokawa \& Yoshida (2018). The accretion rate remained relatively constant at around $\dot{M}_{*} \sim 0.01 \mathrm{M}_{\odot} \mathrm{yr}^{-1}$ for the duration of the simulation $(\sim 250 \mathrm{kyr})$. At this point we now allow the massive Pop III star to transition to a massive black hole seed. Ideally, we would have allowed the Pop III to continue to accrete until it either ran out of nuclear fuel (after $\sim 10^{6} \mathrm{yr}$ ) or reached the GR instability (after reaching a mass of $M_{\text {SMS }} \sim$ $5 \times 10^{5} \mathrm{M}_{\odot}$ ). However, the computational expense in running the simulation at this refinement level is extreme and the physics of massive Pop III star evolution is insufficiently understood to pursue this course. Instead we, prematurely, convert the star particle into a black hole particle in order to study the impact that this change will have on the surrounding material and the accretion on to the black hole.

\subsection{Accretion on to the black hole}

The accretion on to the black hole particle is similar to the accretion mechanism used to accrete on to the star particle. The particle can accrete gas within its accretion radius ( 4 cells) and it can merge with other SMARTSTAR particles. Accretion on to the SMARTSTAR is determined by calculating the flux of gas across the accretion surface.

$\dot{M}=4 \pi \int_{S} \rho v_{r}^{-} r^{2} \mathrm{~d} r$

where $\dot{M}$ is the mass accretion rate, $S$ is the surface over which we integrate, $\rho$ is the density of the cells intersecting the surface, $v_{r}^{-}$is the velocity of cells intersecting the surface and which have negative radial velocities, and $r$ is our surface's radius. The surface, $S$, is the surface of a sphere with radius the accretion radius. As noted above we set the accretion radius to be four cells; we choose to fix this radius independently of the resolution or the mass of the SMARTSTAR. We do this so as to be as accurate as possible when calculating the accretion rate. Any mass travelling radially inwards at a distance of four cells from the SMARTSTAR is taken to be accreted on to the SMARTSTAR - we therefore strive for the maximum possible physical resolution.

As an alternative to directly measuring accretion using the mass flux method described above, we can also calculate the accretion rate on the black hole using the Bondi-Hoyle prescription (Hoyle \& Lyttleton 1939, 1940b,a; Bondi 1952). As described in Krumholz, McKee \& Klein (2004) we use the following approximate formula, which was originally given in this approximate form by Ruffert (1994) and Ruffert \& Arnett (1994):

$\dot{M}=4 \pi \rho_{\infty} r_{\mathrm{BH}}^{2}\left(\lambda^{2} c_{\infty}^{2}+v_{\infty}^{2}\right)^{1 / 2}$,

where $\rho_{\infty}$ is the density of gas at the Bondi-Hoyle radius, $r_{\mathrm{BH}}$ is the Bondi-Hoyle radius, $c_{\infty}$ is the sound speed at infinity (in the host cell in this case), and $v_{\infty}$ is also the relative velocity of the sink particle and the gas in the host cell of the black hole. $\lambda$ is a constant of order unity; we follow Krumholz et al. (2004) in that regard and use $\lambda=\mathrm{e}^{3 / 2} / 4 \sim 1.120$ throughout. While at the high resolution we are able to evolve our simulations at the mass flux approach is more accurate we use the Bondi-Hoyle prescription immediately after jets are launched. We do this to prevent the procedure from calculating spurious accretion rates due to the large mechanical feedback from the jets. After 50 further time-steps the accretion procedure automatically reverts to the mass flux method. We determined this number (50) after careful testing of the mass flux accretion rate against the Bondi-Hoyle accretion rate. We did not employ the Bondi-Hoyle accretion rate for the entire time because we found that during testing (using ENZO and RAMSES) the flux accretion method performed significantly better in several analytic tests, particularly the Shu (1977) collapse test.

The spatial extent of our most refined cells is $\mathrm{d} x \sim 2.5 \times 10^{-4} \mathrm{pc}$ ( $\sim 50 \mathrm{au}$ ). The accretion radius is therefore $R_{\mathrm{acc}} \sim 10^{-3} \mathrm{pc}$. For a $15000 \mathrm{M}_{\odot}$ black hole surrounded by a gaseous medium at approximately $10000 \mathrm{~K}$ the Bondi-Hoyle radius is approximately $r_{\mathrm{BH}} \gtrsim 10^{-2} \mathrm{pc}$ and therefore we are resolving the Bondi-Hoyle radius extremely well at that point. However, as the feedback from the accretion leads to bipolar jets the medium can heat up to close to $10^{6} \mathrm{~K}$ at the edge of the accretion zone leading to a $r_{\mathrm{BH}} \lesssim 10^{-5} \mathrm{pc}$. We are now no longer resolving the Bondi radius and hence we apply the kernel weighting techniques advocated by Krumholz et al. (2004).

In the scenario where the characteristic scale (in this case the Bondi-Hoyle scale) is significantly below the resolution of the simulation it would be erroneous to set the accretion rate derived from a scale significantly beyond the true accretion scale. In this case we apply a kernel-weighted averaging procedure to the accretion rate calculated numerically. In doing this we follow equations 13 and 14 from Krumholz et al. (2004)

$r_{\mathrm{K}}= \begin{cases}\frac{\mathrm{d} x}{4} & \text { if } r_{\mathrm{BH}}<\frac{\mathrm{d} x}{4}, \\ r_{\mathrm{BH}} & \text { if } \frac{\mathrm{d} x}{4} \leq r_{\mathrm{BH}} \leq \frac{r_{\mathrm{acc}}}{2}, \\ \frac{r_{\mathrm{acc}}}{2} & \text { if } r_{\mathrm{BH}}>\frac{r_{\mathrm{acc}}}{2}\end{cases}$

where $r_{\mathrm{K}}$ is the kernel radius and $r_{\mathrm{acc}}$ is the accretion radius (four cells). For all cells then within the accretion radius the kernel weight, $w$, is applied according to

$w \propto \exp \left(-r^{2} / r_{\mathrm{K}}^{2}\right)$,

where the normalization is calculated by computing the sum of the weights.

The accretion on to the star is calculated at each time-step; however, this is likely to be a very noisy metric. To alleviate this to some degree we average the accretion rate over hundreds of timesteps typically corresponding to between 10 and $100 \mathrm{yr}$. The average accretion rate is then used as the actual accretion rate. The accretion rate is added as an attribute to each star and hence a full accretion history of every SMARTSTAR is outputted as part of every snapshot.

\subsection{Feedback from the black hole}

The feedback from an accreting black hole is primarily determined by the radiative efficiency of the disc, $\eta_{\text {disc }}$. $\eta_{\text {disc }}$ is typically set to a value close to 0.1 for a non-rotating black hole. For these simulations we use a value very close to this, $\eta_{\text {disc }}=0.103$, which we derive by explicitly accounting for the spin of the black hole (e.g. Sagdowski et al. 2016)

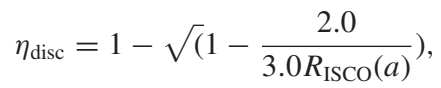

where $a$ is the spin parameter of the black hole, which we set to $a=$ 0.7 , and $R_{\mathrm{ISCO}}$ is a parametrization of the inner most stable orbit given by Abramowicz \& Fragile (2013)

$R_{\mathrm{ISCO}}=R_{G} *\left(3+Z_{2}-\left[\left(3-Z_{1}\right) *\left(3+Z_{1}+2 Z_{2}\right)\right]^{\frac{1}{2}}\right)$,

where $Z_{1}=1+\left(1-a^{2}\right)^{1 / 3}\left((1+a)^{1 / 3}+(1-a)^{1 / 3}\right), Z_{2}=(3 *$ $\left.a^{2}+Z_{1}^{2}\right)^{1 / 2}$, and $R_{G}$ is the gravitational radius, $R_{G}=G M / c^{2}$. 
The accretion rate on to the black hole must now be modified to account for the energy that is returned to the outer medium from the accretion

$\dot{M}_{\mathrm{BH}}=\dot{M} *\left(1-\eta_{\mathrm{disc}}\right)$,

where $\dot{M}_{\mathrm{BH}}$ is now the mass accretion rate on to the black hole while $\dot{M}$ is the numerically determined accretion rate on to the black hole as described in Section 2.3. The feedback from the black hole can now be further decomposed into radiative feedback from the disc and mechanical feedback from a jet component.

\subsubsection{Radiative feedback}

To model the radiative feedback from the black hole we assume a multicolour disc for the accretion disc and then fit a corona with a power law (e.g. Done et al. 2012). We divide the energy radiated equally between the multicolour disc and the power-law component. The radiative feedback within ENZO is modelled using the ray tracing module MORAY (Wise \& Abel 2011), which discretizes the radiation into a set of finite energy bins which are then transported outwards from the black hole particle. We split the radiation into five energy bins from infrared up to hard X-rays. The energy bins used are 2.0, 12.8, 19.1, 217.3, and $5190 \mathrm{eV}$ with the actual value of the luminosity at each time-step determined by the accretion rate at that time-step. The fractional energy in each energy bin is then determined by the accretion rate on to the black hole and the mass of the black hole. For super-Eddington adjustments to the radiative feedback we employ the fit from Madau, Haardt \& Dotti (2014), who themselves use Sagdowski (2009) to derive the fit. In this case the luminosity is calculated as

$\frac{L}{L_{\mathrm{E}}}=A(a)\left(\frac{0.985}{\dot{M}_{\mathrm{E}} / \dot{M}_{\mathrm{BH}}+B(a)}+\frac{0.015}{\dot{M}_{\mathrm{E}} / \dot{M}_{\mathrm{BH}}+C(a)}\right)$,

where the functions $A, B$, and $C$ scale with the spin of the black hole, $a$, as

$A(a)=(0.9663-0.9292 a)^{-0.5639}$

$B(a)=(4.627-4.445 a)^{-0.5524}$

$C(a)=(827.3-718.1 a)^{-0.7060}$

and $\dot{M}_{\mathrm{E}}$ is the Eddington mass accretion rate. The luminosity per solar mass is adjusted in this case compared to the thin disc model but the energy bins and energy fraction per bin remain unchanged. In essence the radiative efficiency is reduced, as expected.

For the cases considered here we limit the radiative feedback component to non-ionizing radiation only - i.e. we use the first two energy bins of our model only. We do this for three reasons. First, the simulations are computationally expensive and in order to reduce the computational expense we limit the radiative feedback to being optically thin and below the ionization potential of hydrogen. Secondly, the fraction of energy emitted as ionizing radiation falls off rapidly as the accretion rate decreases and by considering only the infrared and Lyman-Werner components we are none the less still capturing the bulk of the radiative processes. Finally, in this work we are primarily interested in investigating the impact of mechanical feedback (i.e. jets) on the ability of seed black holes to accrete effectively and hence neglecting the ionizing radiation component allows us to do that. A full treatment of the radiative feedback will be considered in an upcoming study. Appendix A contains a detailed discussion of the procedure used in determining the spectral energy distribution (SED) of the black hole simulated in this paper.

\subsubsection{Mechanical feedback}

Microphysical models of the physics of accretion discs have shown that bipolar jets produced predominantly by the tangling of magnetic field lines are a robust feature of super-Eddington accretion. Jets appear to also be present at low accretion rates, most frequently when the accretion rates fall below $10^{-3} M_{\text {Edd }}$ (e.g. Merloni \& Heinz 2008; Sa̧dowski et al. 2016, and references therein), but in this paper we want to focus on the effects of jets launched during superEddington phases; therefore, we do not initiate jets for accretion rates below the Eddington rate and instead all of the feedback is radiative in that case (below the ionization threshold of hydrogen as discussed above).

To calculate how much energy is mechanical output in the superEddington regime, we again follow the models of Sadowski et al. (2016; equations 42 through 46). In this case the total jet luminosity is given by

$L_{\mathrm{jet}}=\eta_{\mathrm{jet}} \dot{M}_{\mathrm{BH}} c^{2}$,

where $\eta_{\text {jet }}$ is the jet efficiency factor given by (Sagdowski et al. 2016)

$\eta_{\text {jet }}=1.3 a^{2}$.

This efficiency assumes maximum efficiency of the jet, where we have assumed a 'MAD' value of 1, making this an upper limit to the jet efficiency (Sajdowski et al. 2016). An additional complication in modelling jets is that jets are an inherently relativistic phenomenon and their launch speed is close to the speed of light. Furthermore, the jets are launched on scales close to $R_{G}$, which is far below the resolution of our simulations. Therefore, modelling both the speed and the initial launch radius of the jet is beyond the capabilities of ENZO. Hence we 'mass load' the jet (e.g Ciotti \& Ostriker 2001; Dubois et al. 2012) by adding additional mass to the jet and by reducing the speed of the jet. This accounts for the assumption that the speed of the jet will diminish as the jet entrains mass on its way from the black hole. The mass-loading factor, $\beta_{\text {jet }}$, is defined as

$\beta_{\text {jet }}=\frac{\dot{M}_{\text {jet }}}{\dot{M}_{\mathrm{BH}}}$

where $\dot{M}_{\text {jet }}$ is the amount of material ejected by the jet per unit time and again $\dot{M}_{\mathrm{BH}}$ is the mass projected to accrete on to the black hole surface. We now define the jet 'kinetic' power, $\mathrm{KE}_{\text {jet }}$, as in Kim et al. (2011) and equate it to the luminosity of the jet using conservation of energy to write

$\mathrm{KE}_{\mathrm{jet}}=\frac{1}{2} \dot{M}_{\mathrm{jet}} v_{\mathrm{jet}}^{2}$

$\mathrm{KE}_{\text {jet }}=L_{\text {jet }}$.

We can then equate the mass-loading factor, $\beta_{\text {jet }}$, with the velocity of the jet, $v_{\text {jet }}$, and write

$\beta_{\text {jet }}=2 \eta_{\text {jet }} \frac{c^{2}}{v_{\text {jet }}^{2}}$

and we see that the mass loading of the jet and the velocity of the jet are degenerate, as expected. Typically, in numerical simulations $v_{\text {jet }}$ is set to be much less than $c$. For example setting $v_{\text {jet }}=0.1 c$ 
gives $\beta_{\text {jet }}=127.4$ while $v_{\text {jet }}=0.01 c$ gives $\beta_{\text {jet }}=12740$. In both cases $\beta_{\text {jet }} \gg 1$.

Attempting to mass-load the jet by factors of up to $10^{5}$ can be problematic as there may not be enough mass in the surrounding cells to do so. In this, rather common, case we adjust the mass accretion rate on to the black hole so that the black hole can effectively only accrete for a fraction of the current time-step. The fraction is calculated so there is sufficient mass to load the jet. Ideally, we would like to decrease the time-step of the simulation such that the total mass required for a single accretion + feedback episode, i.e. $M_{\mathrm{tot}}=\Delta M_{\mathrm{BH}}+\Delta M_{\text {jet }}=\left(1+\beta_{\text {jet }}\right) * M_{\mathrm{BH}}$, is less than the total mass available in the accretion region, i.e. $<M_{\text {acc }}$ where $\Delta M_{\mathrm{BH}}=\dot{M}_{\mathrm{BH}} \Delta t$. Practically, this will make the time-step unaffordably short, particularly in the super-Eddington accretion regime, where both the amount of mass removed from the grid and the total energy to be returned to the grid will be high. Instead we decrease the accretion time in this subgrid manner. We do this by introducing a factor, $\epsilon_{t}$, which operates on the accretion rate modifying both the actual accretion rate found for the black hole and the resulting jet ejection rate. $\epsilon_{t}$ is calculated as

$\epsilon_{t}=\min \left(1.0, \frac{\dot{M}_{\mathrm{acc}}}{\dot{M}} \frac{1.0}{1-\eta_{\mathrm{disc}}} \frac{1.0}{1+\beta_{\mathrm{jet}}}\right)$,

where $\dot{M}_{\text {acc }}$ is the maximum possible accretion rate, i.e. the total mass in the accretion sphere divided by the time-step. The above equation ensures mass conservation within the subgrid algorithm with $\epsilon_{t}$ fixed to be always less than one. A further consequence of this approach is that the ejected mass is very similar in all cases independent of the speed of the jet. Consider the following.

$\dot{M}_{\text {jet }}=\beta_{\text {jet }}\left(1-\eta_{\text {disc }}\right) \dot{M} \frac{\dot{M}_{\text {acc }}}{\dot{M}} \frac{1.0}{1-\eta_{\text {disc }}} \frac{1.0}{1+\beta_{\text {jet }}}$

$\dot{M}_{\mathrm{jet}}=\frac{\dot{M}_{\mathrm{acc}}}{\frac{1}{\beta_{\mathrm{jet}}}+1}$

$\dot{M}_{\text {jet }} \sim \dot{M}_{\text {acc }} \quad$ for $\beta_{\text {jet }} \gg 1$

Hence, we have found that the mass ejected by the jet will be close to, but always less than, the mass in the surrounding accretion sphere. This is expected since we need $\epsilon_{t}$ to be such that there is always sufficient mass available to mass-load the jet. Clearly, this is not as good as allowing the time-step to drop to the required value but recall that $\epsilon_{t}$ is only less than unity in the case where no mass is available for accretion and feedback at that time-step. It is therefore a practical approach to a resolution-limited problem. In practice we find that for the vast majority of the time $\epsilon_{t}=1$.

Now that the algorithm for determining the mass of the jet and the speed of the jet has been determined, it remains to describe how the jets are launched within the simulation. In this regard we follow both Kim et al. (2011) and Dubois et al. (2012). Kim et al. (2011) use 'supercells' within the ENZO grid hierarchy to launch the jets, effectively adding mass and velocity to cells on the outer edge of a cone to launch the jet. As recommended by Dubois et al. (2012) we insert the jet at the maximum resolution and over the minimum number of cells as possible so as to have the jet as collimated as possible. Typical jets are observed to be less than $1000 R_{G}$ in radius (e.g. Doeleman et al. 2012) and so well below the resolution of our simulations - hence we insert the jet over a limited number of cells (26). These 26 cells are the cells that are immediately surrounding the black hole (i.e. $3^{3}-1=26$ ). [See also fig. 2 from Kim et al. (2011) for a pictorial representation of the 'supercells'. Note that Kim et al. 2011 injected velocity into cells that were further from the black hole, in neighbour of neighbour cells and so over 98 cells $\left(5^{3}\right.$ $\left.-2^{3}=98\right)$.] We inject cells close to the black hole with velocity, directed along the angular momentum vector and antiparallel to it, which results in bipolar jets that are as highly collimated as our resolution allows. The effect of this is to make the jet denser relative to spreading the jet over a larger number of cells - the typical density of the jets launched in our simulations is $\rho_{\text {jet }} \sim 10^{10} \mathrm{~cm}^{-3}$.

\subsection{Simulation realizations}

In order to test different mass-loading values we select three different jet velocities. The speed of the jet impacts the mass-loading value through equation (18). Jets due to super-Eddington accretion rates are launched at relativistic speeds; however, modelling relativistic jets is computationally challenging and so mass-loading the jet is often preferred. In this study we examine three different jet launching speeds: (1) $6000 \mathrm{~km} \mathrm{~s}^{-1}$, (2) $30000 \mathrm{~km} \mathrm{~s}^{-1}$, and (3) $100000 \mathrm{~km} \mathrm{~s}^{-1}$. These speeds correspond to $0.018 c, 0.1 c$, and $0.33 c$. As outlined in equation (22), the mass ejected by the jet during each outburst is similar in all cases. Therefore, the difference between each realization is effectively only in the speed of the jets and hence the momentum and energy of the jets in each realization. As we will see, all three realizations result in similar effective accretion rates regardless of the jet speed chosen.

\section{RESULTS}

Our goal in this study is to examine the earliest stages of seed black hole growth immediately after the SMS or massive Pop III collapses into a black hole. As was found in the SMS simulations in $\mathrm{R} 18 \mathrm{~b}$ the accretion rate on to the massive Pop III star after $250 \mathrm{kyr}$ is approximately $0.01 \mathrm{M}_{\odot} \mathrm{yr}^{-1}$. However, feedback from an accreting black hole is much more powerful than that of an SMS or a massive Pop III star due to the significantly enhanced compactness of the black hole. We here investigate primarily the impact of the mechanical feedback on future accretion.

In Fig. 1 we plot the mass accretion rate (right-hand panel) and the total mass accreted by the black hole (left-hand panel). In the lefthand panel we plot the mass accretion rate over the first $100000 \mathrm{yr}$ after the seed black hole forms for three different values of jet velocity. The $x$-axis runs from $-50 \mathrm{kyr}$ to $120 \mathrm{kyr}$. The negative times indicate the time for when the object was in its SMS phase. In this phase the feedback is modelled using radiative feedback from non-ionizing radiation only, consistent with current theories of SMS evolution (Hosokawa et al. 2013a; R18b). At $T=0$ years the SMS collapses into a black hole and the initial accretion rates are super-Eddington which in turn drive powerful jets. In the BH phase feedback is modelled using both non-ionizing radiation (similar to the SMS phase) and mechanical feedback from jets. Therefore, the main difference in feedback between the negative and positive times is the mechanical feedback.

The impact of the jets is clearly visible. Jet events are immediately followed by periods of very low accretion before gas cools and falls back to the centre of the potential again. The periods of high accretion, which generate the jet events, are episodic, with periods of between a few kiloyears and $20 \mathrm{kyr}$ approximately. The dynamical time of high-density gas in these simulations is approximately $10 \mathrm{kyr}$ at a radius of $1 \mathrm{pc}$, which is consistent with the cycles of accretion found here.

The green line, jets with launch velocities of $0.1 c$, shows a slightly different behaviour to the two other realizations. In the case of the jet with velocities of $0.1 c$ initial periods of super-Eddington 
accretion are followed by a very large drop in accretion before it gradually rebuilds again. After the first two periods of superEddington accretion the gas never again falls in at sufficient rates to generate jets and instead the accretion remains relatively steady at approximately $10^{-4} \mathrm{M}_{\odot} \mathrm{yr}^{-1}$. It is interesting that the jets with both higher and lower launch speeds show qualitatively different behaviour and it highlights the variability of such systems. After the gas is expelled from the central object it does fall back again, on approximately the dynamical time, but it need not necessarily fall back at the same rates. Also, this behaviour is shown to not be directly correlated with the launch speed.

We also plot the results of a lower resolution run (dashed red line) for the $0.33 c$ jets. The resolution is reduced by a factor of 4, down from 20 levels of refinement to 18 levels of refinement. The simulations show broadly the same behaviour but the detailed dynamics of the gas are different due to the lower resolution of this run. In this case the jet is spread over a larger radius, with the result showing that accretion is suppressed for a longer period of time compared to the higher resolution case.

In the right-hand panel we plot the total mass accreted by the black hole against time. We calculate the effective accretion rate simply by taking the initial black hole mass from the final black hole mass divided by the time. The effective mass accretion rate is less than $10^{-4} \mathrm{M}_{\odot} \mathrm{yr}^{-1}$ in all cases. This is two orders of magnitude below the accretion rate on to the massive Pop III star immediately prior to collapse into the black hole. Over the course of $100000 \mathrm{yr}$ the black holes grow by only approximately $10 \mathrm{M}_{\odot}$ in each realization. If accretion at this rate were to continue, the black holes would increase their mass by only one order of magnitude over one billion years. They would grow to become intermediate-mass black holes with masses of $M_{\mathrm{BH}} \sim 10^{5} \mathrm{M}_{\odot}$ in the early Universe. By way of comparison the mean accretion rate during active phases is $1.3 \times 10^{-4} \mathrm{M}_{\odot} \mathrm{yr}^{-1}$. We define active phases as those for which the black hole is accreting at more than $10^{-5} \mathrm{M}_{\odot} \mathrm{yr}^{-1}$.

In Fig. 2 we plot projections of the number density in a $10 \mathrm{pc}$ cube surrounding the black hole of the simulation of the black hole with jet velocities of $0.018 c$. Similar projections of the simulations with $0.1 c$ and $0.33 c$ can be found in Appendix B. Overplotted on top of the density field is the velocity field with directional arrows. The length of the arrows is proportional to the value of the velocity at that point. The plots are made near the start and near the end of the simulation. Initially, in the left-hand panel, we see the black hole (marked in green) surrounded by high-density gas with strong outflows due to jet events. The strong outflows are noticeable, at this scale, only from the longer-than-average velocity line. The jets are highly successful at disrupting accretion on to the black hole but the impact of the jet is local. The jets are not able to globally influence the halo. As the simulations proceed gas is driven out of the very central regions and must fall back in order for accretion to pick up again. The dynamical time for this system is determined by both the gravitational potential of the black hole, at small radii, and the self-gravity of the gas at larger radii. We calculate the effective dynamical time as

$t_{\mathrm{dyn}}=\left(\frac{1}{t_{\mathrm{bh}}^{2}}+\frac{1}{t_{\mathrm{ff}}^{2}}\right)^{-0.5}$

where $t_{\mathrm{ff}}$ is the self-gravity of the gas given by $t_{\mathrm{ff}}=\sqrt{\frac{3}{32 G \rho}}$ and $t_{\mathrm{bh}}$ is the dynamical time of the gas within the potential of the black hole given by $t_{\mathrm{bh}}=\sqrt{\frac{R^{3}}{G * M_{\mathrm{BH}}}}$ and $\rho$ is the gas density, $G$ is the gravitational constant, $R$ is the radius from the black hole, and $M_{\mathrm{BH}}$ is the mass of the black hole. For our system this corresponds to $10 \mathrm{kyr}$ at a radial distance of a few parsecs from the centre of the halo down to less than $1 \mathrm{kyr}$ for the highest density gas within $0.1 \mathrm{pc}$ of the centre. In each case what is immediately noticeable is that the outflows from the jets have little or no effect on gas outside of approximately $1 \mathrm{pc}$. In each realization there is no fingerprint from the jet activity at scales larger than this even though the jets are launched with velocities of up to $100000 \mathrm{~km} \mathrm{~s}^{-1}$. The inflow from the gas is easily able to overwhelm the jet momentum, so while the jets are able to effectively shut off accretion in the immediate radius of the black hole they have no effect on the gas at scales of a parsec or larger.

In Fig. 3 we 'zoom-in' to the region immediately surrounding the black hole in the simulation with jet velocities of $6000 \mathrm{~km} \mathrm{~s}^{-1}$. To illustrate the impact of the accretion events and the jet launching events we focus on visualizing the behaviour of the black hole between $30 \mathrm{kyr}$ and $40 \mathrm{kyr}$ after the formation of the black hole. At this point the black hole is rapidly accreting material at slightly sub-Eddington accretion rates (top left- and top right-hand panels of Fig. 3; see also Fig. 1). As the accretion rate continues to increase, it eventually exceeds the Eddington rate at approximately $37 \mathrm{kyr}$, resulting in an outflow and a decrease in the accretion rate (bottom left- and bottom right-hand panels of Fig. 3)

In Figs 4, 5, and 6 we quantify the projection plots by taking ray profiles for different times during the course of the simulation. The $1 \mathrm{D}$ ray profiles are created by profiling the gas properties perpendicular to the angular momentum vector of the gas (i.e. in the plane of the accretion disc). Focusing first on the simulation with jet velocities of $6000 \mathrm{~km} \mathrm{~s}^{-1}$ (i.e. Fig. 4) we see that jet events reduce the density of the gas by up to a few orders of magnitude out to a distance of approximately $0.1 \mathrm{pc}$ following at outflow. The low-density gas that is left behind is superheated to a temperature of $10^{6} \mathrm{~K}$ and the gas is also fully ionized out to approximately $1 \mathrm{pc}$. The gas receives positive outward momentum from the jet events. The positive radial velocities given to the gas (as seen in the upper right-hand panel as the green line) sweeps gas away from the black hole. This reduces the density of gas in the vicinity of the black hole and shuts off accretion. It is therefore primarily the momentum given to the jets that shuts off the accretion mechanics.

None the less the gas is quickly able to recover and fall back into the centre of the potential and in a little over $10 \mathrm{kyr}$ the gas has reached sufficient density that the black hole can accrete at very high rates and can indeed again exceed the Eddington rate (see Fig. 1). This is supported by the fact that the dynamical times for the gas between $1 \mathrm{pc}$ and $10 \mathrm{pc}$ is approximately $10 \mathrm{kyr}$.

Figs 5 and 6 show both qualitatively and quantitatively similar results. Periods of super-Eddington accretion launch jets, which drive high-density gas out from the centre of the halo. The density of the gas surrounding the black hole is temporarily reduced by several orders of magnitude out to a distance of approximately $0.1 \mathrm{pc}$. However, the gas quickly falls back to the centre of the potential well, where again gas can be accreted at high rates driving another jet event. We note here also that the ray profiles are examining, on average, the highest density gas in the plane of the accretion disc. Outside of the plane of the disc the density can be much lower following an accretion event.

\section{SUMMARY AND DISCUSSION}

In this study we examine the impact of super-Eddington accretion and feedback on the growth rate of (supermassive) black hole seeds. 

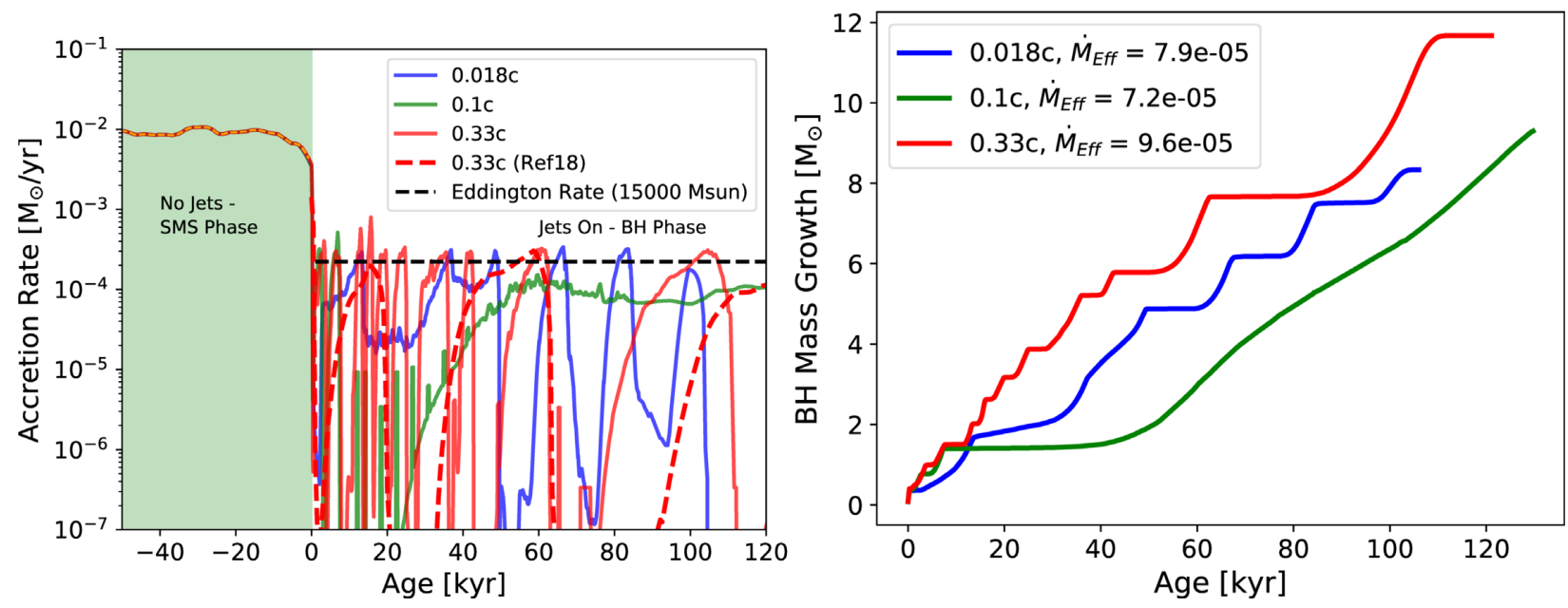

Figure 1. Left-hand panel: mass accretion rates on to the black hole for different mass-loading prescriptions. The $x$-axis runs from $-50 \mathrm{kyr}$ to $120 \mathrm{kyr}$. The black hole forms at $T=0 \mathrm{yr}$. The negative time-scale represents the SMS phase of the object. In this phase there is only non-ionizing radiative feedback and no jets. Jets turn on once the object collapses to a $\mathrm{BH}$ at $T=0$. The appearance of the jets signals a severe shift in the accretion history of the object. All simulations show that the jets effectively limit accretion to below the Eddington rate. Once accretion exceeds the Eddington limit, the jets turn on disrupting the accretion flow. Differences between the impact of different prescriptions are clearly visible but similarities exist. The dynamical time for the highest density gas in the centre of the halo is a few thousand years and so gas falls back to the centre on this time-scale. This is evident from the spikes for each prescription. The dashed red line is a lower resolution simulation using jets with launch speeds of $0.33 c$. Qualitatively the behaviour is similar to the higher resolution runs. Right-hand panel: the mass growth of each seed black hole. The initial mass of each seed is identical, $M_{\text {seed }}=15904 \mathrm{M}_{\odot}$. The effective accretion rate on to each seed is calculated over $100000 \mathrm{yr}$ and found to be almost $\dot{M} \sim 10^{-4} \mathrm{M}_{\odot} \mathrm{yr}^{-1}$ in each case. This results in a mass increase of roughly $10 \mathrm{M}_{\odot}$ in each case over the first $100000 \mathrm{yr}$ of the seed black hole's existence.
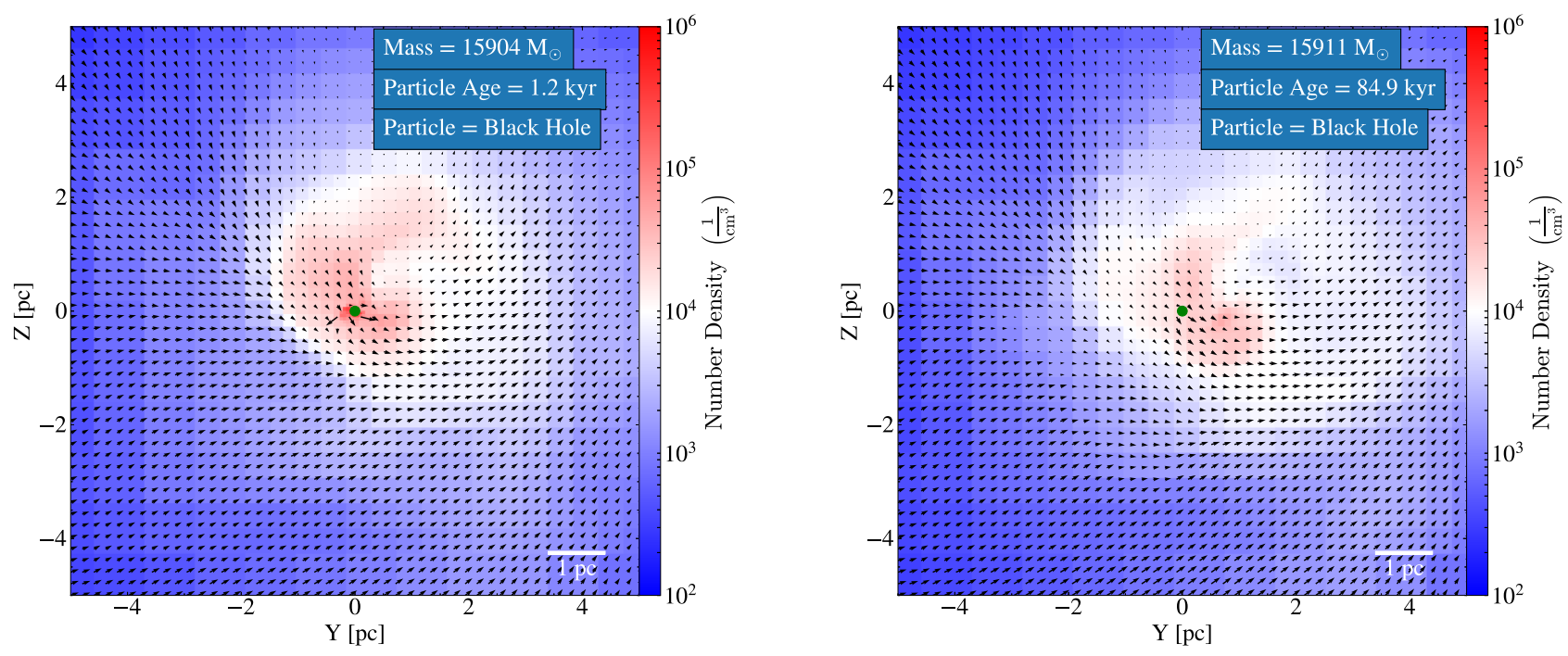

Figure 2. The number density of the gas in a $10 \mathrm{pc}$ volume surrounding the black hole (marked in green). The velocity of jets in this projection is $6000 \mathrm{~km} \mathrm{~s}^{-1}$. The velocity of the jets is marked with arrows to give the direction. As the accretion rate exceeds the Eddington rate bipolar jets are launched from around the black hole (e.g. in the left-hand panel). We make the visualizations near the start of the black hole evolution and near the end. In the left-hand panel the black hole has just released an outflow - which can be seen as the two longer-than-average velocity arrows. However, at this scale of a few parsecs the impact of the jets is very mild. The jets have a strong local effect, as we will see, but globally they have little effect on the halo. The impact of the jets is clearly visible in Fig. 3 where we zoom in on the region surrounding the black hole. Note that the radial extent of the black hole has been greatly exaggerated for this plot.

In order to create $\mathrm{SMSs}$, rapid accretion on to a proto-star is required, with accretion rates of close to $0.1 \mathrm{M}_{\odot} \mathrm{yr}^{-1}$ thought to be necessary to inflate the envelope surrounding a proto-star and create an SMS. If such accretion rates can be maintained after the collapse of the SMS, then super-Eddington accretion on to the seed black hole may be expected.

We here investigate exactly this scenario. We use the selfconsistent SMS/massive Pop III simulations of R18b as a starting point for our simulations. The starting point is a massive Pop III star accreting at approximately $0.01 \mathrm{M}_{\odot} \mathrm{yr}^{-1}$ with a final stellar mass of $15,904 \mathrm{M}_{\odot}$. To examine the subsequent accretion on to a seed black hole, we artificially collapse the massive Pop III star and create a direct-collapse black hole seed. No supernova or other feedback from the massive Pop III star is modelled to precede the formation of a black hole. After black hole formation we model radiative feedback, below the ionization threshold, and mechanical feedback 


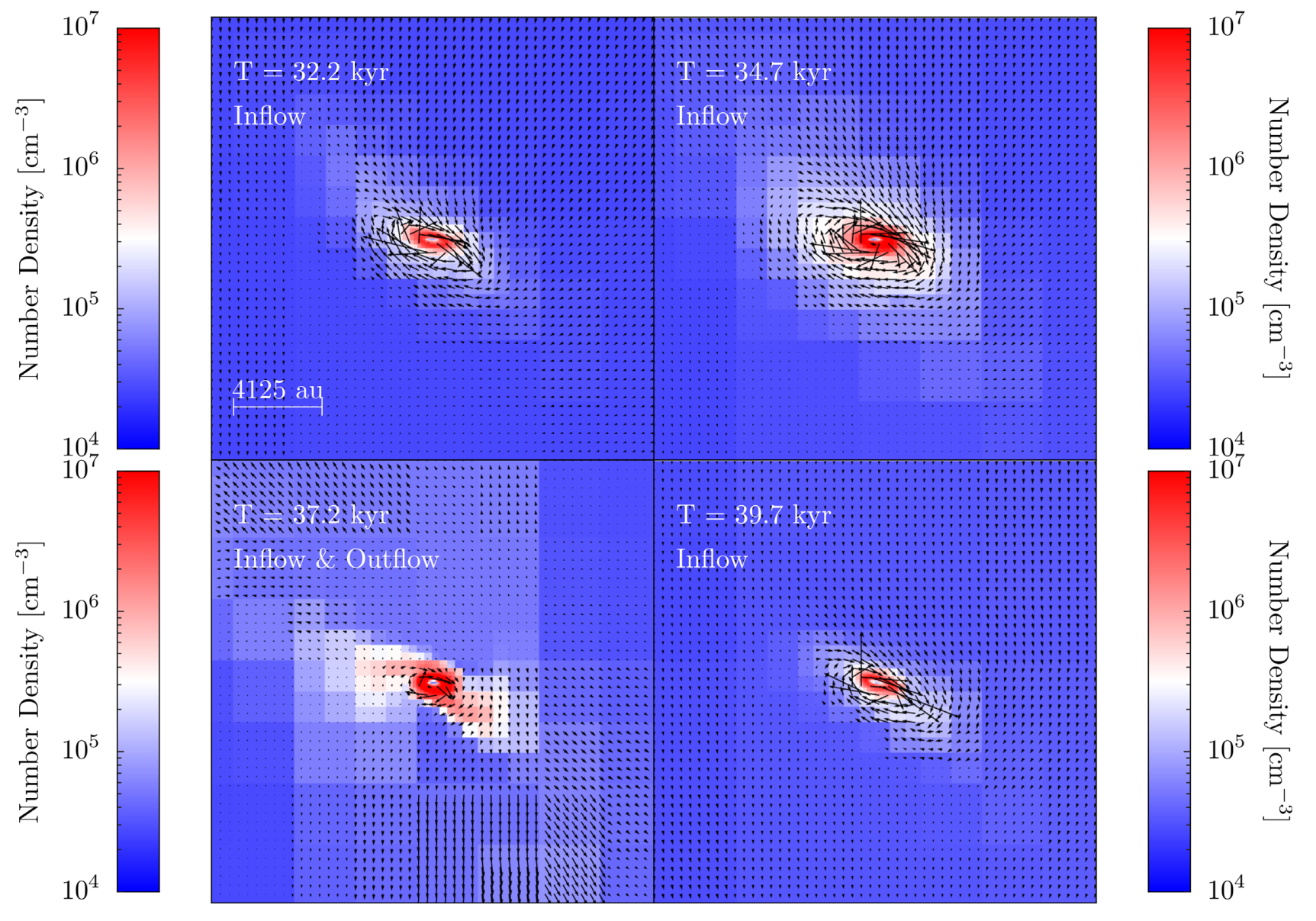

Figure 3. A 'zoom-in' projection on to a period of accretion (inflow) followed by a jet event (outflow). The projections are made for the simulation with jet outflows of $6000 \mathrm{~km} \mathrm{~s}^{-1}$ between approximately $30 \mathrm{kyr}$ and $40 \mathrm{kyr}$ after the formation of the black hole. The scale line shown in the top left-hand panel gives a scale of $4125 \mathrm{au}$. Each panel covers a region of $0.1 \mathrm{pc}$ on the side. A super-Eddington outflow occurs approximately at $T \sim 37 \mathrm{kyr}$ (see also Fig. 1) following accretion above the Eddington limit. Further accretion is then able to continue following the outflow.

in the form of bipolar jets for super-Eddington accretion events. We therefore focus almost entirely on the impact that jet feedback has on the growth of the black hole in this study. While radiative feedback could in principle regulate accretion to the Eddington rate, we do not model this here. We instead choose to examine the impact of super-Eddington accretion rates and the potential negative feedback associated with the jets driven by these extreme accretion rates. As discussed in Section 2.4.2 we launch bipolar jets at the maximum resolution our set-up allows. The jets are launched by spreading the velocity of the jets equally over 27 cells just outside the accretion radius of our black hole. This corresponds to a physical resolution for the jet radius of approximately $300 \mathrm{au}$. This is still much coarser than the radius at which jets are launched by black holes of comparable sizes and hence our jets may still not be sufficiently collimated. This remains an inherent limitation of these simulations.

We modelled the bipolar jets using three different mass-loading values. We modelled jets with velocities of $6000(0.018 c), 30000$ $(0.1 c)$, and $100000 \mathrm{~km} \mathrm{~s}^{-1}(0.33 c)$. The results were qualitatively similar in each case. Periods of super-Eddington accretion generate violent bipolar jets that suppress accretion by many orders of magnitude. The gas surrounding the black hole is successfully evacuated due to the positive radial velocity of the jets and the gas must wait on the free-fall time before being available for accretion again. However, the jets are unable to break out of the very central region of the halo. The inflow is easily able to overwhelm the outflows. We see no impact from the jets at scales greater than approximately $1 \mathrm{pc}$ and so the jets are found to be an inherently local phenomenon with no global impact for black holes of this mass $\left(\sim 15000 \mathrm{M}_{\odot}\right)$. The local impact of the jets is significant and the jets successfully shut off the super-Eddington accretion that launched them initially, leading to periods of low accretion immediately after jet launching, giving rise to episodic accretion as in the case of radiative feedback from light seeds (Milosavljević et al. 2009). These periods of inactivity lead to effective accretion a factor of a few below the Eddington rate.

While a full resolution study of the results presented here is outside the scope of this work we did run the $0.33 c$ simulation at 4 times lower resolution (see Fig. 1). We found that qualitatively the results followed the same pattern of periods of low accretion following a jet event followed by a return to super-Eddington accretion followed by a further jet event. In a future study we will explore a larger parameter space to quantitatively access the impact of resolution. However, tentatively our results do suggest that with increased resolution of the jet such a system may be able to sustain super-Eddington accretion more efficiently.

The Eddington ratio, $\lambda$, can be used to describe accretion rates that are below Eddington. In Fig. 7 we plot the growth rate of a black hole seed starting from the initial mass of the seed black hole studied here $\left(M_{\text {init }}=15904 \mathrm{M}_{\odot}\right)$. The growth rate for a black hole 


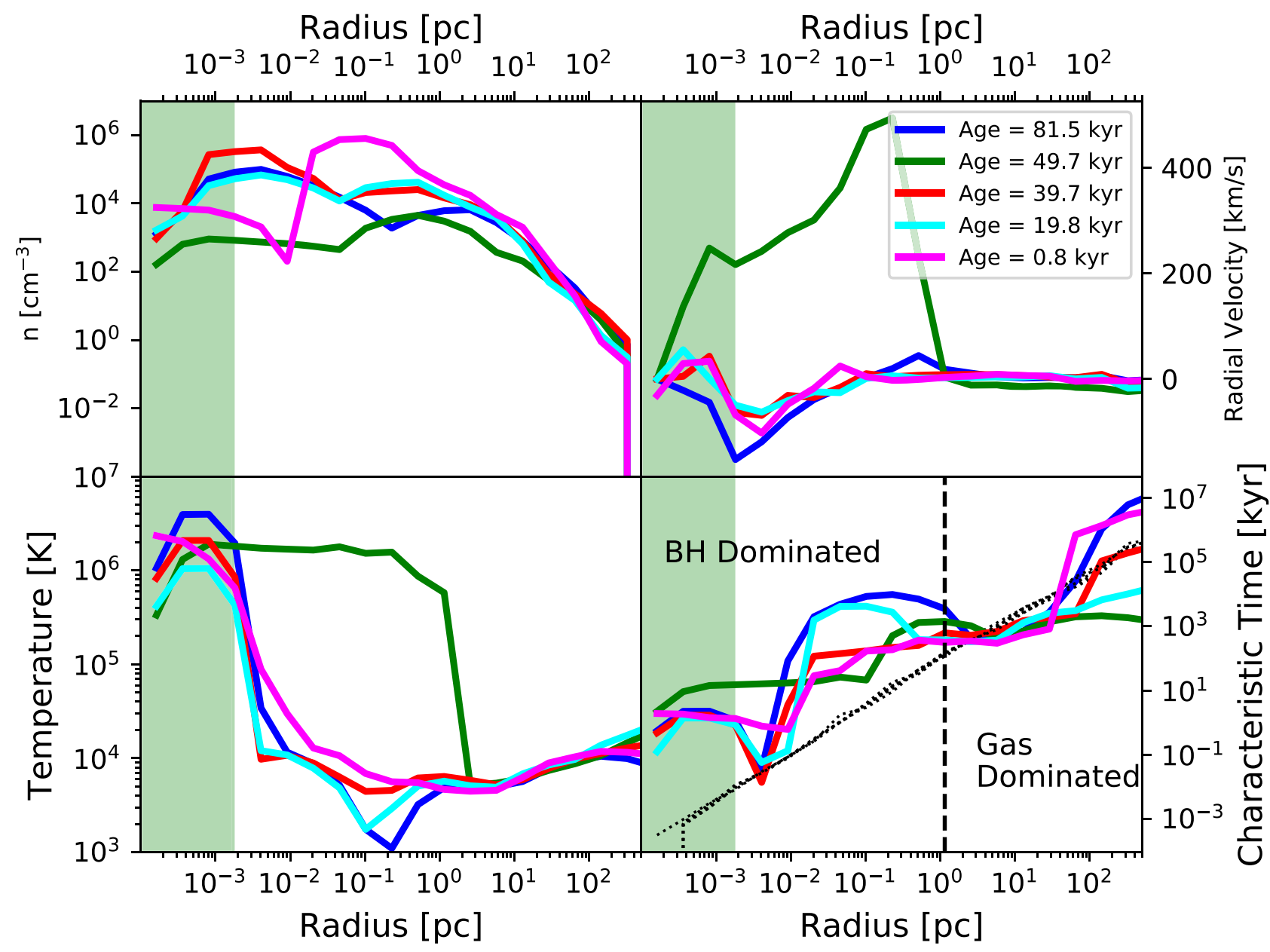

Figure 4. Ray profile plots of the number density, temperature, electron fraction, and 'characteristic' time of the gas at times between seed black hole formation and the end of the simulation after $100000 \mathrm{yr}$. The characteristic time plot contains both the dynamical time (black dotted lines) and the cooling time of the gas (coloured lines). The green shaded region on the extreme left of each panel is the accretion zone of the SMARTSTAR particle (i.e. the black hole). Values inside this region should be treated with caution as the gas is accreted from within this region and we advise readers not to draw conclusions from values inside the accretion zone. The profiles are 1D profiles, perpendicular to the angular momentum vector (i.e. through the accretion disc). The impact of the jets is most strikingly seen in the green line in this figure (compare the time to Fig. 1). At age $\sim 49.7 \mathrm{kyr}$ the accretion rate exceeds the Eddington rate, driving a jet and mass outflow at $6000 \mathrm{~km} \mathrm{~s}^{-1}$. The temperature rapidly increases to over $10^{6} \mathrm{~K}$ out to almost $1 \mathrm{pc}$ and the gas is strongly ionized. The flow of the gas is clearly seen in the radial velocity profile plot (top right). Within the disc the gas is accreting on to the disc and black hole but further out the outflow, as a result of the jet, is clearly visible. The dynamical time of the gas increases linearly with radius with the cooling time-scales always longer than the dynamical time-scales. At radii less that approximately $1 \mathrm{pc}$ the dynamical time-scale is dominated by the black hole. Outside of that time the gas is dominated by the self-gravity of the infalling gas. The dynamical time for the gas within $1 \mathrm{pc}$ is less than $10 \mathrm{kyr}$ and so the gas is able fall back to the centre on approximately this time-scale.

seed is given by

$M(t)=M\left(t_{0}\right) \exp \left(t / t_{\text {Edd }}\right)$,

where $M(t)$ is the mass after time $t, M\left(t_{0}\right)$ is the initial seed mass at $t_{0}=0$, and $t_{\text {Edd }}$ is the Eddington (or Salpeter) time given by

$t_{\mathrm{Edd}}=\frac{\sigma_{\mathrm{T}} \eta_{\mathrm{disc}} c}{4 \pi G m_{\mathrm{p}}} \approx \eta_{\mathrm{disc}} 5 \times 10^{8} \mathrm{yr}$,

with $\eta_{\text {disc }}$ the usual disc efficiency with canonical value 0.1 . In this case the Eddington time for black hole is approximately $50 \mathrm{Myr}$. The growth of the black hole is then often counted in the number of efolding times required to reach a predetermined mass. In Fig. 7 we plot as dashed vertical lines the efolding times for the seed black hole modelled here. The exponential nature of black hole growth means that initially growth is quite sedentary and it only picks up as larger efolding times are reached. The $\lambda$ factor is used to describe growth rates that are below Eddington. In this study we found that the bipolar jets reduce growth to a factor of a few below the Eddington rate. We have also plotted these curves in our plot assuming that both $\eta$ and $\lambda$ are time independent. Growth rates that are a factor of 2 or more below Eddington have their mass, after 11 efolding times, reduced by more than two orders of magnitude.

However, the black hole growth over longer times will likely be determined more by the dynamics of the host halo and its ability to merge with other haloes, which may promote more efficient growth (e.g. Valiante et al. 2016; Pezzulli et al. 2017). If the black hole host halo is part of a number of major mergers this will undoubtedly promote more efficient accretion of material. Star formation in the surrounding gas will act to diminish growth by consuming available gas, although we see no evidence of star formation in our simulations in the first $100000 \mathrm{yr}$ after black hole formation.

If stars start to form and gas is enriched (internally or externally), the gas distribution will change. On the one hand, gas will be 


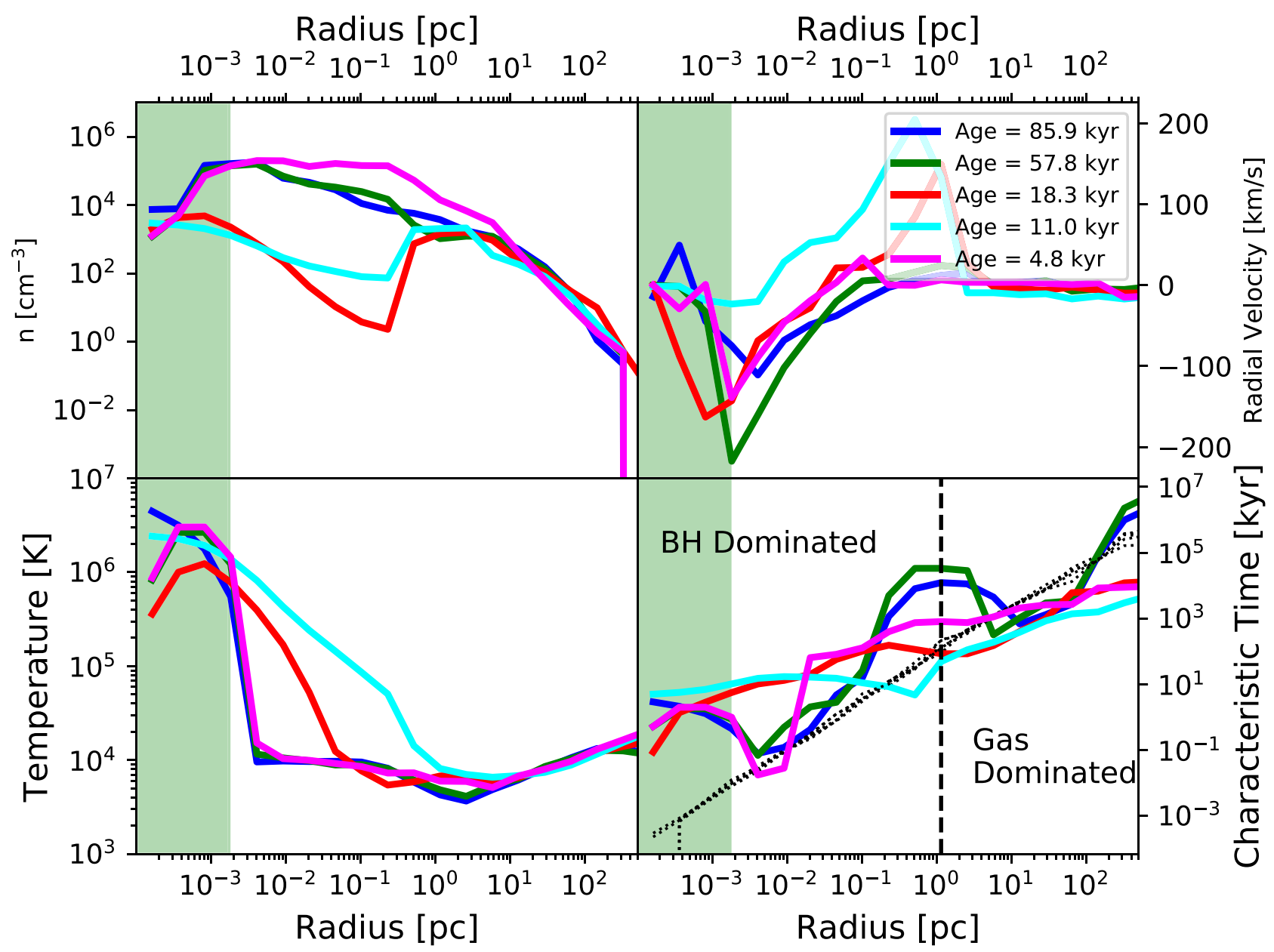

Figure 5. The same as Fig. 4 for the $30000 \mathrm{~km} \mathrm{~s}^{-1}$ simulation. In this case we show the effect of a strong initial jet at age $\sim 11$ kyr which drives gas away from the centre of the halo (see the large outflow velocity at $R \sim 1 \mathrm{pc}$ ). None the less, the gas recovers and can fall back into the centre, again reactivating accretion, within a few kiloyears.

consumed in star formation. On the other hand, gas will cool more easily, fragment, and generate regions of high and low density. If the cooling gas possesses angular momentum, it will settle in a disc from which the jet can more easily escape without damaging the surroundings if the jet propagates orthogonally to the disc (Cielo et al. 2018). If none of this happens and the halo grows by accreting metal-free intergalactic gas, perhaps the central density will be so high that the jet cannot do any damage even in its immediate surroundings and in this case super-Eddington accretion may well be possible for extended durations. Modelling the growth of seed black holes over several tens of megayears will require significantly more computational power and also the identification of realistic target haloes that form a seed in an environment that is favourable to rapid accretion at rates at or above the Eddington rate as the black hole grows. Such simulations are likely to be possible in the near future.

The idealized nature of our set-up and the relatively short time for which we are able to evolve our simulations mean that we are unable to provide more detailed information on the subsequent growth of the black hole. None the less, accretion on to seed black holes formed from the direct collapse of a massive Pop III star is much more efficient than the accretion on to Pop III remnant black holes
$\left(M_{\text {init }} \sim 100 \mathrm{M}_{\odot}\right.$; e.g. Smith et al. 2018) that have initial accretion rates many orders of magnitude below the Eddington rate.

As noted above we also do not model the impact of ionizing radiation on the accretion rate of the seed black hole. The impact of ionizing radiation has been modelled by numerous other authors (e.g. Milosavljević et al. 2009; Park \& Ricotti 2012; Park, Wise \& Bogdanović 2017; Sugimura et al. 2018) in 1D, 2D, and 3D simulations. The conclusions are broadly similar - isotropic radiation feedback has a strongly negative impact on black hole growth. More idealized models (Inayoshi et al. 2016; Sugimura et al. 2017) have shown circumstances where the impact of radiative feedback can be overcome but these models have not yet translated over to more general realizations. Either way the broad conclusions are that radiative feedback, in general, leads to a strongly negative impact on the accretion rate. Added to this scenario the efficient removal of angular momentum of gas from the system is another hurdle that must be understood (e.g. Krumholz, McKee \& Klein 2005; Sugimura et al. 2018). Similar to the results found here, the general conclusion is that feedback (be it radiative or mechanical) has a detrimental impact on black hole growth, and more detailed investigations of the black hole environment, conditions, and circumstances to achieve maximal growth are still required. 


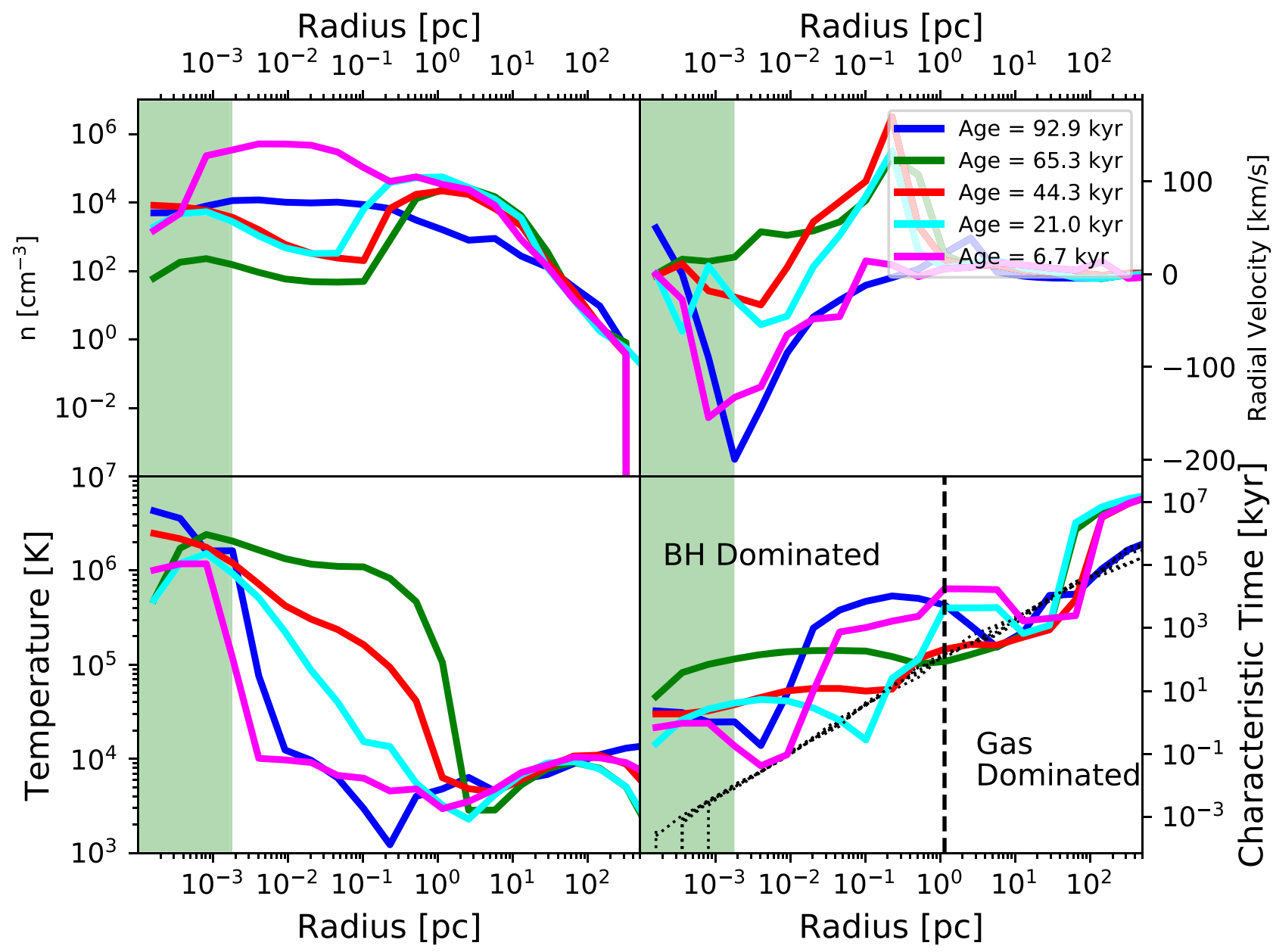

Figure 6. The same as Figs 4 and 5 for the $100000 \mathrm{~km} \mathrm{~s}^{-1}$ simulation. A similar pattern is observed. Jets are able to effectively drive gas away from the black hole severely suppressing growth. The gas recovery time can be as low as a few hundred years for the highest densities.

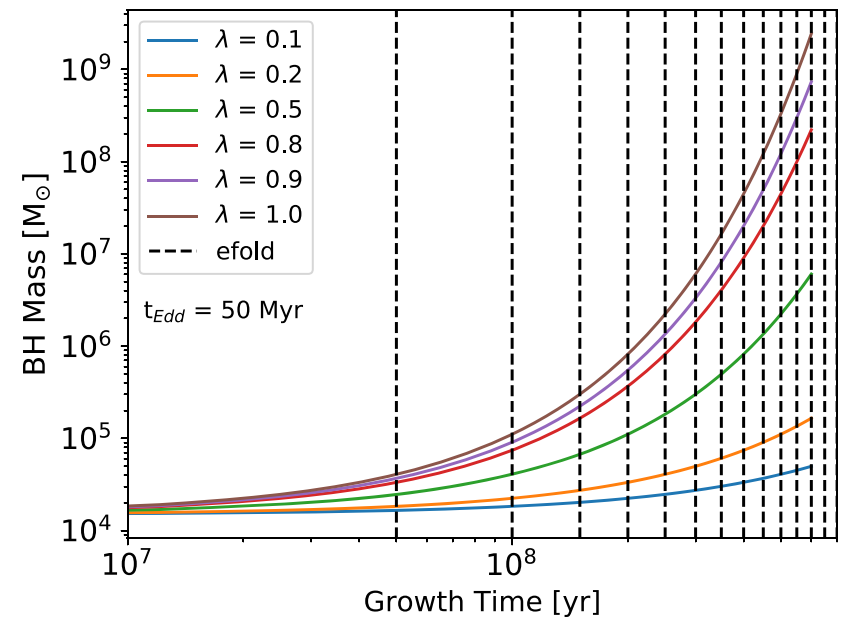

Figure 7. The Eddington-limited growth rate of massive black holes for different values of the Eddington ratio, $\lambda$. The Eddington ratio is defined by $\lambda=\dot{M}_{\mathrm{BH}} / \dot{M}_{\text {Edd }}$. Values of $\lambda$ that deviate even moderately from 1.0 have significantly reduced growth. We find that mechanical feedback led to an effective accretion rate approximately 0.25 times the canonical Eddington rate and hence an expected growth rate close to the orange line shown. The vertical dashed lines are the efolding times determined from the Eddington time.

\section{CONCLUSIONS}

Mechanical feedback from bipolar jets is able to quickly evacuate high-density gas from the accreting black hole once the accretion rate exceeds the canonical Eddington rate. However, the gas quickly recombines and falls back towards the centre of the potential on the freefall time of the system. The impact of the jet outflows is local to the immediate surroundings of the black hole. We find that the bipolar jets are unable to break out of the halo and indeed have no impact on scales greater than approximately $1 \mathrm{pc}$. None the less, the jets have a significant impact on the black hole accretion rate. The effective accretion rate, taking into account periods of high accretion and intervening periods of quiescence, is reduced by a factor of a few below the Eddington accretion rate for at least the first $100000 \mathrm{yr}$ after the formation of the black hole.

As an example, a reduction in the black hole accretion rate of a factor of 2 below the Eddington rate, if it were to remain at this level over the first 500 hundred million years of the black hole growth, would reduce the mass of the black hole by a factor of at least 20 . The mass after 10 efolding times would be between $10^{5} \mathrm{M}_{\odot}$ and $10^{6} \mathrm{M}_{\odot}$ assuming the black hole continues to accrete at half the Eddington rate. Therefore, direct-collapse black holes born into atomic cooling haloes will require external influences (e.g. rapid major mergers with other haloes) to promote efficient accretion and reach $\mathrm{SMBH}$ masses within a few hundred million years. Further 
investigation of rapidly growing direct-collapse host haloes will be required in the coming years to test the growth prospects of massive black hole seeds in realistic haloes.

\section{ACKNOWLEDGEMENTS}

JAR acknowledges the support of the EU Commission through the Marie Skłodowska-Curie Grant - 'SMARTSTARS' - grant number 699941. MV, AL, and MT acknowledge funding from the European Research Council under the European Community's Seventh Framework Programme (FP7/2007-2013 Grant Agreement no. 614199, project 'BLACK'). RSB acknowledges funding from the Centre National de la Recherche Scientifique (CNRS) on grant ANR-16-CE31-0011. Computations described in this work were performed using the publicly available ENZO code (http: //enzo-project.org), which is the product of a collaborative effort of many independent scientists from numerous institutions around the world. Their commitment to open science has helped make this work possible. The freely available astrophysical analysis code YT (Turk et al. 2011) was used to construct numerous plots within this paper. The authors would like to extend their gratitude to Matt Turk et al. for an excellent software package. JAR would like to thank Lydia Heck and all of the support staff involved with Durham's COSMA4 and DiRAC's COSMA5 systems for their technical support. This work was supported by the Science and Technology Facilities Council (grant numbers ST/L00075X/1 and RF040365). This work used the DiRAC Data Centric system at Durham University, operated by the Institute for Computational Cosmology on behalf of the STFC DiRAC HPC Facility (www.dirac.ac.uk). This equipment was funded by BIS National E-infrastructure capital grant ST/K00042X/1, STFC capital grant ST/H008519/1, and STFC DiRAC Operations grant ST/K003267/1 and Durham University. DiRAC is part of the National E-Infrastructure.

\section{REFERENCES}

Abel T., Anninos P., Zhang Y., Norman M. L., 1997, New Astron., 2, 181 Abramowicz M. A., Fragile P. C., 2013, Living Rev. Relativ., 16, 1

Abramowicz M. A., Czerny B., Lasota J. P., Szuszkiewicz E., 1988, ApJ, 332,646

Alvarez M. A., Wise J. H., Abel T., 2009, ApJ, 701, L133

Anninos W. Y., Norman M. J., 1994, ApJ, 429, 434

Begelman M. C., Volonteri M., Rees M. J., 2006, MNRAS, 370, 289

Begelman M. C., Rossi E. M., Armitage P. J., 2008, MNRAS, 387, 1649

Bondi H., 1952, MNRAS, 112, 195

Bromm V., Loeb A., 2003, ApJ, 596, 34

Bryan G. L., Norman M. L., O'Shea B. W., Abel T., Wise J. H., Turk M. J., The Enzo Collaboration, 2014, ApJS, 211, 19

Chandrasekhar S., 1964, ApJ, 140, 417

Chen P., Wise J. H., Norman M. L., Xu H., O’Shea B. W., 2014, ApJ, 795 , 144

Chon S., Hosokawa T., Yoshida N., 2018, MNRAS, 475, 4104, preprint ()

Cielo S., Bieri R., Volonteri M., Wagner A. Y., Dubois Y., 2018, MNRAS, 477, 1336

Ciotti L., Ostriker J. P., 2001, ApJ, 551, 131

Coppola C. M., Longo S., Capitelli M., Palla F., Galli D., 2011, ApJS, 193 , 7

Coppola C. M., D’Introno R., Galli D., Tennyson J., Longo S., 2012, ApJS, 199,16

Devecchi B., Volonteri M., 2009, ApJ, 694, 302

Dijkstra M., Haiman Z., Mesinger A., Wyithe J. S. B., 2008, MNRAS, 391, 1961

Dijkstra M., Ferrara A., Mesinger A., 2014, MNRAS, 442, 2036

Doeleman S. S. et al., 2012, Science, 338, 355
Done C., Gierliński M., Kubota A., 2007, A\&AR, 15, 1

Done C., Davis S. W., Jin C., Blaes O., Ward M., 2012, MNRAS, 420, 1848

Du P. et al., 2018, ApJ, 856, 6

Dubois Y., Pichon C., Haehnelt M., Kimm T., Slyz A., Devriendt J., Pogosyan D., 2012, MNRAS, 423, 3616

Eisenstein D. J., Loeb A., 1995, ApJ, 443, 11

Fan X. et al., 2006, AJ, 131, 1203

Fernandez R., Bryan G. L., Haiman Z., Li M., 2014, MNRAS, 439, 3798

Freitag M., Gürkan M. A., Rasio F. A., 2006, MNRAS, 368, 141

Glover S. C. O., 2015a, MNRAS, 451, 2082

Glover S. C. O., 2015b, MNRAS, 453, 2901

Glover S. C. O., Abel T., 2008, MNRAS, 388, 1627

Glover S. C. O., Jappsen A.-K., 2007, ApJ, 666, 1

Glover S. C. O., Savin D. W., 2009, MNRAS, 393, 911

Gürkan M. A., Freitag M., Rasio F. A., 2004, ApJ, 604, 632

Gürkan M. A., Fregeau J. M., Rasio F. A., 2006, ApJ, 640, L39

Habouzit M., Volonteri M., Dubois Y., 2017, MNRAS, 468, 3935

Haemmerlé L., Woods T. E., Klessen R. S., Heger A., Whalen D. J., 2018a, MNRAS, 474, 2757, preprint ()

Haemmerlé L., Woods T. E., Klessen R. S., Heger A., Whalen D. J., 2018b, MNRAS, 474, 2757

Hahn O., Abel T., 2011, MNRAS, 415, 2101

Heger A., Fryer C. L., Woosley S. E., Langer N., Hartmann D. H., 2003, ApJ, 591, 288

Hirano S., Hosokawa T., Yoshida N., Kuiper R., 2017, Science, 357, 1375

Hockney R. W., Eastwood J. W., 1988, Computer Simulation Using Particles. Hilger, Bristol

Hosokawa T., Omukai K., Yorke H. W., 2013a, ApJ, 778, 178

Hosokawa T., Yorke H. W., Inayoshi K., Omukai K., Yoshida N., 2013b, ApJ, 778, 178

Hoyle F., Lyttleton R. A., 1939, Proc. Camb. Philos. Soc., 35, 405

Hoyle F., Lyttleton R. A., 1940a, Proc. Camb. Philos. Soc., 36, 325

Hoyle F., Lyttleton R. A., 1940b, Proc. Camb. Philos. Soc., 36, 424

Inayoshi K., Omukai K., 2012, MNRAS, 422, 2539

Inayoshi K., Omukai K., Tasker E., 2014, MNRAS, 445, L109

Inayoshi K., Visbal E., Kashiyama K., 2015, MNRAS, 453, 1692

Inayoshi K., Haiman Z., Ostriker J. P., 2016, MNRAS, 459, 3738

Jeon M., Pawlik A. H., Greif T. H., Glover S. C. O., Bromm V., Milosavljević M., Klessen R. S., 2012, ApJ, 754, 34

Jiang Y. F., Stone J., Davis S. W., 2017, preprint (arXiv:1709.02845)

Johnson J. L., Bromm V., 2007, MNRAS, 374, 1557

Katz H., Sijacki D., Haehnelt M. G., 2015, MNRAS, 451, 2352

Kim J. h., Wise J. H., Alvarez M. A., Abel T., 2011, ApJ, 738, 54

Kitsionas S., Whitworth A. P., 2002, MNRAS, 330, 129

Krumholz M. R., McKee C. F., Klein R. I., 2004, ApJ, 611, 399

Krumholz M. R., McKee C. F., Klein R. I., 2005, ApJ, 618, 757

Latif M. A., Bovino S., Grassi T., Schleicher D. R. G., Spaans M., 2015, MNRAS, 446, 3163

Lupi A., Haardt F., Dotti M., Fiacconi D., Mayer L., Madau P., 2016, MNRAS, 456, 2993

Madau P., Haardt F., Dotti M., 2014, ApJ, 784, L38

Mayer L., Kazantzidis S., Escala A., Callegari S., 2010, Nature, 466, 1082

Mayer L., Fiacconi D., Bonoli S., Quinn T., Roškar R., Shen S., Wadsley J., 2015, ApJ, 810, 51

Merloni A., Heinz S., 2008, MNRAS, 388, 1011

Milosavljević M., Couch S. M., Bromm V., 2009, ApJ, 696, L146

Mitsuda K. et al., 1984, PASJ, 36, 741

Mortlock D. J. et al., 2011, Nature, 474, 616

O'Shea B. W., Abel T., Whalen D., Norman M. L., 2005, ApJ, 628, L5

O’Shea B. W., Wise J. H., Xu H., Norman M. L., 2015, ApJ, 807, L12

Omukai K., Schneider R., Haiman Z., 2008, ApJ, 686, 801

Pacucci F., Volonteri M., Ferrara A., 2015, MNRAS, 452, 1922

Park K., Ricotti M., 2012, ApJ, 747, 9

Park K., Wise J. H., Bogdanović T., 2017, ApJ, 847, 70

Pezzulli E., Valiante R., Schneider R., 2016, MNRAS, 458, 3047

Pezzulli E., Volonteri M., Schneider R., Valiante R., 2017, MNRAS, 471, 589 
Portegies Zwart S. F., Baumgardt H., Hut P., Makino J., McMillan S. L. W., 2004, Nature, 428, 724

Regan J. A., Downes T. P., 2018a, MNRAS, 475, 4636

Regan J. A., Downes T. P., 2018b, MNRAS, 478, 5037(R18b)

Regan J. A., Haehnelt M. G., 2009a, MNRAS, 396, 343

Regan J. A., Haehnelt M. G., 2009b, MNRAS, 393, 858

Regan J. A., Johansson P. H., Wise J. H., 2015, MNRAS, 449, 3766

Regan J. A., Visbal E., Wise J. H., Haiman Z., Johansson P. H., Bryan G. L., 2017, Nat. Astron., 1, 0075

Ruffert M., 1994, ApJ, 427, 342

Ruffert M., Arnett D., 1994, ApJ, 427, 351

Sakurai Y., Inayoshi K., Haiman Z., 2016a, MNRAS, 461, 4496

Sakurai Y., Vorobyov E. I., Hosokawa T., Yoshida N., Omukai K., Yorke H. W., 2016b, MNRAS, 459, 1137

Schauer A. T. P., Regan J., Glover S. C. O., Klessen R. S., 2017, MNRAS, 471,4878

Schleicher D. R. G., Palla F., Ferrara A., Galli D., Latif M., 2013, A\&A, 558, A59

Shu F. H., 1977, ApJ, 214, 488

Smith B. D. et al., 2017, MNRAS, 466, 2217

Smith B. D., Regan J. A., Downes T. P., Norman M. L., O'Shea B. W., Wise J. H., 2018, MNRAS, 480, 3762

Stone J. M., Norman M. L., 1992a, ApJS, 80, 753

Stone J. M., Norman M. L., 1992b, ApJS, 80, 791

Sugimura K., Omukai K., Inoue A. K., 2014, MNRAS, 445, 544

Sugimura K., Hosokawa T., Yajima H., Omukai K., 2017, MNRAS, 469, 62

Sugimura K., Hosokawa T., Yajima H., Inayoshi K., Omukai K., 2018, MNRAS, 478, 3961

Sa̧dowski A., 2009, ApJS, 183, 171

Sạdowski A., Narayan R., 2016, MNRAS, 456, 3929

Sạdowski A., Narayan R., McKinney J. C., Tchekhovskoy A., 2014, MNRAS, 439, 503

Sagdowski A., Lasota J. P., Abramowicz M. A., Narayan R., 2016, MNRAS, 456,3915

Tanaka T. L., Li M., 2014, MNRAS, 439, 1092

Tang J.-J. et al., 2019, MNRAS, 484, 2575

Toyouchi D., Hosokawa T., Sugimura K., Nakatani R., Kuiper R., 2019, MNRAS, 483, 2031

Tseliakhovich D., Hirata C., 2010, Phys. Rev. D, 82, 083520

Turk M. J., Smith B. D., Oishi J. S., Skory S., Skillman S. W., Abel T., Norman M. L., 2011, ApJS, 192, 9

Umeda H., Hosokawa T., Omukai K., Yoshida N., 2016, ApJ, 830, L34

Valiante R., Schneider R., Volonteri M., Omukai K., 2016, MNRAS, 457, 3356

Visbal E., Haiman Z., Bryan G. L., 2014, MNRAS, 445, 1056

Wang J.-M., Chen Y.-M., Hu C., 2006, ApJ, 637, L85

Whalen D., Abel T., Norman M. L., 2004, ApJ, 610, 14

Wise J. H., Abel T., 2011, MNRAS, 414, 3458

Wolcott-Green J., Haiman Z., 2012, MNRAS, 425, L51

Woods T. E., Heger A., Whalen D. J., Haemmerlé L., Klessen R. S., 2017, ApJ, 842, L6

Woosley S. E., Heger A., Weaver T. A., 2002, Rev. Mod. Phys., 74, 1015

Xu H., Wise J. H., Norman M. L., 2013, ApJ, 773, 83

\section{APPENDIX A: BLACK HOLE SPECTRAL ENERGY DISTRIBUTION}

In this paper we model radiation from the black holes below the ionization threshold of hydrogen only. We omit the impact of ionizing feedback so as to concentrate solely on the mechanical feedback from the jets. None the less our implementation is set up to calculate the energy spectrum from the black hole accretion disc and we elucidate that methodology here for the interested reader.

The calculation is based on the assumption of a multicolour blackbody disc surrounded by a hot corona. The implementation within ENZO is based on a lookup table that tabulates the SED

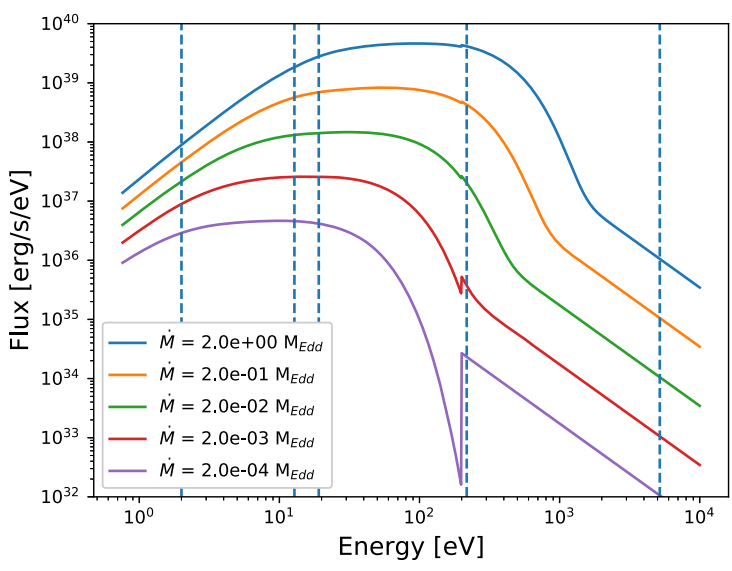

Figure A1. An example of the SED for the black holes used in this study. The mass of the black hole in this case is $M_{\mathrm{BH}}=15000 \mathrm{M}_{\odot}$. The SED changes with both the black hole mass and the accretion rate. We discretize the radiation into five bins $(2.0,12.8,19.1,217.3$, and $5190 \mathrm{eV})$. The bins are marked with dashed lines on the plot. The SED is composed of a multicolour disc and a power law for the high-energy hard X-ray part of the spectrum. The contribution from the high-energy component of the spectrum falls off significantly as the accretion rate on to the black holes decreases.

based on the mass of the black hole and the black hole accretion rate. The masses range from $1 \mathrm{M}_{\odot}$ up to $10^{9} \mathrm{M}_{\odot}$ with mass accretion rates running from $10^{-6} \mathrm{M}_{\odot} \mathrm{yr}^{-1}$ up to $1^{3} \mathrm{M}_{\odot} \mathrm{yr}^{-1}$. We begin by examining the multicolour disc (MCD) component (Mitsuda et al. 1984; Done, Gierliński \& Kubota 2007). The model assumes that the local emission from the disc is Planckian with a temperature profile $T(r) \propto r^{-3 / 4}$. The flux from the black hole can then be written as

$F_{\mathrm{MCD}}=\int_{R_{\text {outer }}}^{R_{\text {inner }}} 2 \pi R B(E, T) \mathrm{d} R$,

where $F_{\mathrm{MCD}}$ is the flux emanating from the MCD, $R_{\text {inner }}$ and $R_{\text {outer }}$ are the inner and outer boundaries of the accretion disc, $B(E, T)$ is the Planck function, and $T$ is the temperature. $R_{\text {inner }}$ is set to be equal to the inner most stable circular orbit (i.e. $R_{\text {inner }}=R_{\text {isco }}=6 R_{\text {sh }}=$ $\left.6 G M / c^{2}\right)$, where $R_{\mathrm{sh}}$ is the Schwartzchild radius $\left(G M / c^{2}\right) . R_{\text {outer }}$ is set to be 1000 times $R_{\mathrm{sh}}$. The inner disc temperature, $T_{\text {inner }}$, is given by

$T_{\text {inner }}=\left(\frac{3 G M_{\mathrm{BH}} \dot{M}_{\mathrm{BH}}}{8 \pi R_{\text {inner }}^{3} \sigma_{\mathrm{SB}}}\right)^{0.25}$,

where $M_{\mathrm{BH}}$ is the mass of the black hole, $\dot{M}_{\mathrm{BH}}$ is the accretion rate on to the black holes, and $\sigma_{\mathrm{SB}}$ is the Stefan-Boltzmann constant. The disc temperature, $T(R)$, is then found by applying the scaling relation $T(r) \propto r^{-3 / 4}$. Once the flux for the MCD is found, what remains is to add the contribution from the corona surrounding the accretion disc.

For modelling the contribution from the corona we apply a power law with spectral power index of $\Gamma=-1.7$. The black hole normalization in this case can be written as

$\mathrm{BH}_{\text {norm }}=\frac{(1+\Gamma) L_{\mathrm{BH}}}{E_{\text {end }}^{\Gamma+1}-E_{\text {start }}^{\Gamma+1}}$,

where $L_{\mathrm{BH}}=\eta \dot{M}_{\mathrm{BH}} c^{2}, E_{\text {start }}$ and $E_{\text {end }}$ are the limits of the energy over which the corona applies, and $\eta$ is the radiative efficiency. $E_{\text {start }}=200 \mathrm{eV}$ and $E_{\text {end }}=10000 \mathrm{eV}$. The spectral energy component from the corona, $F_{\mathrm{C}}$, can then be written as

$F_{\mathrm{C}}=\mathrm{BH}_{\text {norm }} E^{\Gamma}$, 
where $E$ is the energy range of the (high-energy) corona. The total energy contribution from the black hole is split equally between the MCD and corona and each component is multiplied by 0.5 in the actual calculation.

In Fig. A1 we plot the SED for a selection of black hole accretion rates for a black hole with a mass of $15000 \mathrm{M}_{\odot}$. A $15000 \mathrm{M}_{\odot}$ the black hole experiencing super-Eddington accretion will have a peak in the SED of approximately $100 \mathrm{eV}$ and sustained emission into the hard X-ray. As the accretion rate deteriorates (e.g. after an outflow) the peak in the SED regresses to lower energies with most of the energy lying below the ionization threshold for hydrogen once the accretion rate drops below approximately $10^{-3} \dot{M}_{\text {Edd }}$.

\section{APPENDIX B: VISUALIZATIONS OF THE SIMULATIONS WITH JETS OF VELOCITY O.1c AND 0.33c}

The projections in a $10 \mathrm{pc}$ cube surrounding the black hole are shown in Figs B1 and B2. Qualitatively they are similar to Fig. 2.
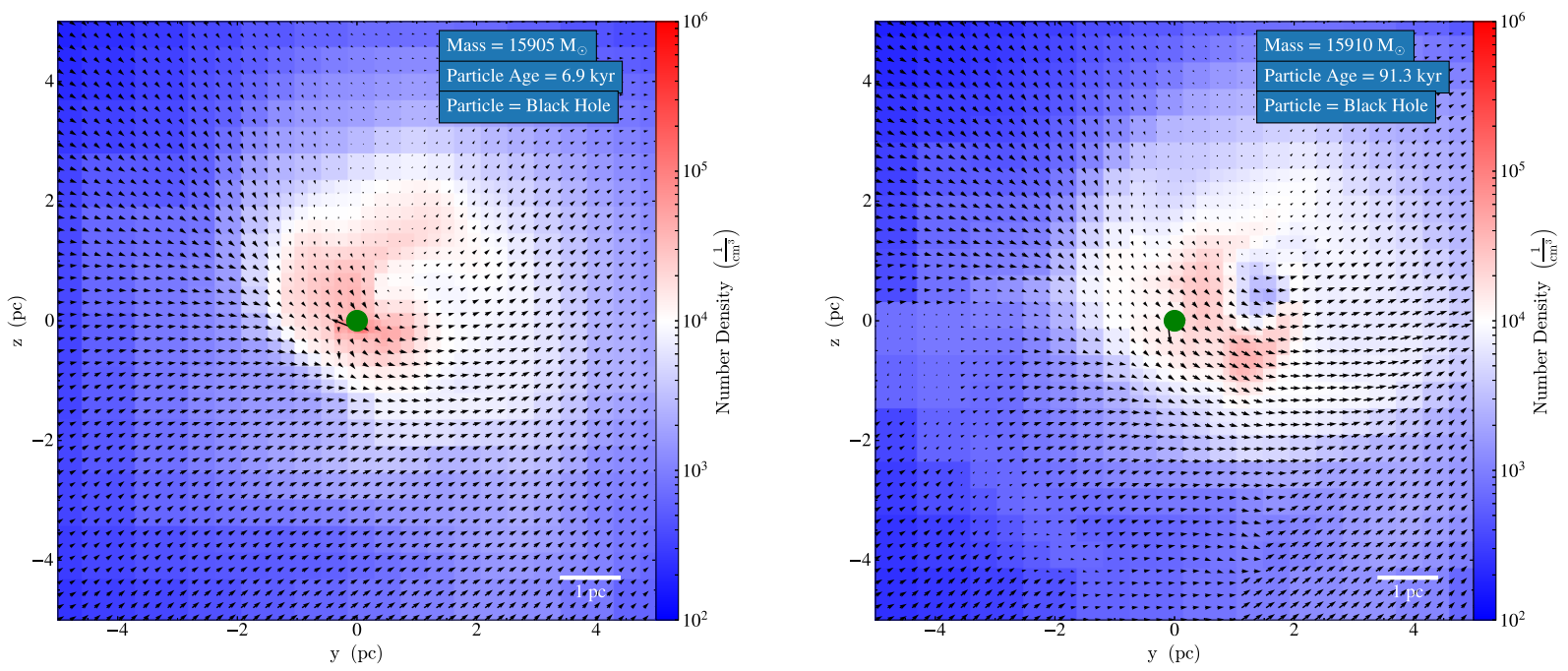

Figure B1. The same as Fig. 2 except for the simulation with jet velocities of $30000 \mathrm{~km} \mathrm{~s}^{-1}$. This simulation suffers an initial large drop in accretion rates after the first few jets are launched. The accretion rates then settle down to a more or less constant rate of $\dot{M}_{\mathrm{BH}} \sim 10^{-4} \mathrm{M}_{\odot} \mathrm{yr}^{-1}$.
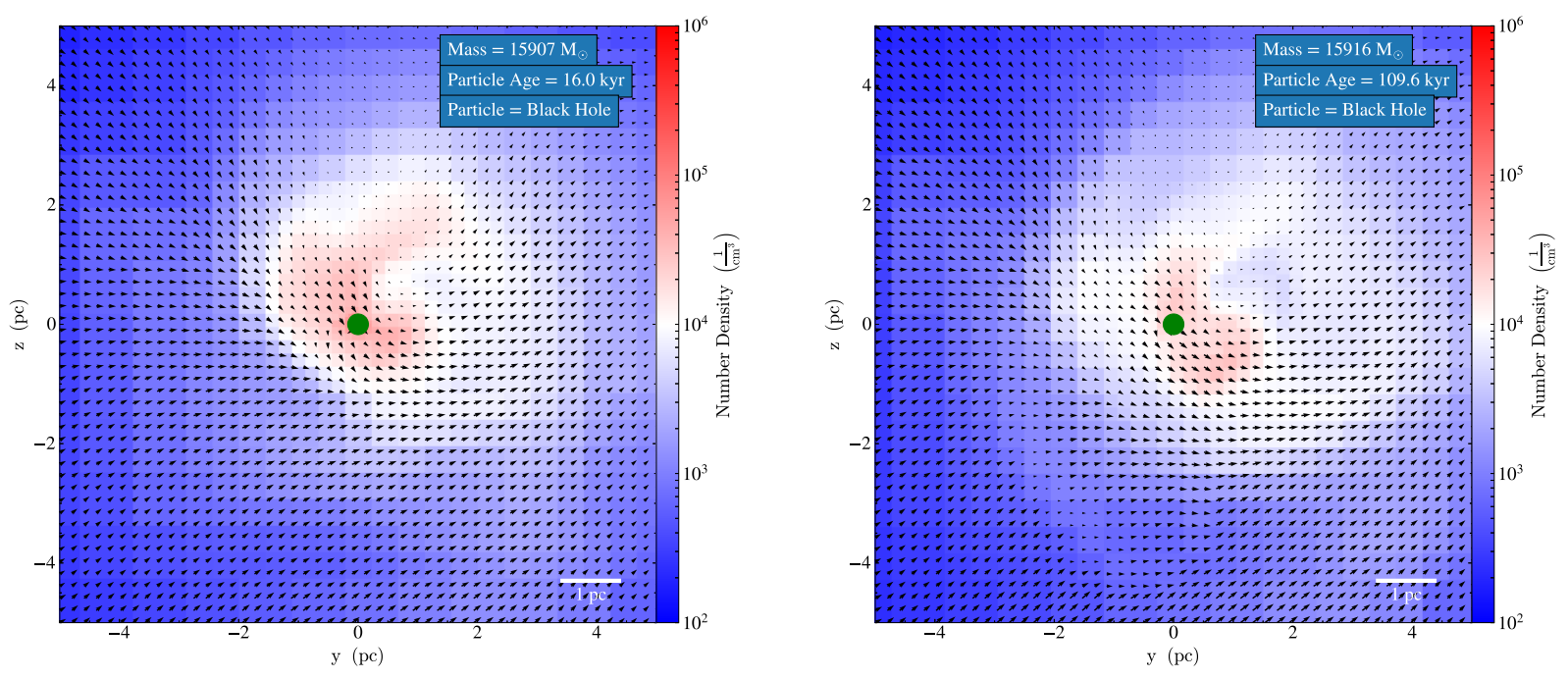

Figure B2. The same as Figs 2 and B1 except for the simulation with jet velocities of $100000 \mathrm{~km} \mathrm{~s}^{-1}$. This is the realization with the highest jet velocities and hence the lowest mass loading. None the less, even with these extremely high outflow velocities there is little, if any, impact from the jets at the parsec scale. The impact of the jets is only seen at sub-parsec scales but they can significantly hinder accretion. 